\title{
Mutual Coupling Effects for Radar Cross Section (RCS) of a Series-Fed Dipole Antenna Array
}

\author{
H. L. Sneha, Hema Singh, and R. M. Jha \\ Computational Electromagnetics Laboratory, Aerospace Electronics and Systems Division, National Aerospace Laboratories \\ (CSIR-NAL), Bangalore 560017, India
}

Correspondence should be addressed to Hema Singh, ishihema30@gmail.com

Received 18 June 2012; Accepted 11 August 2012

Academic Editor: Hon Tat Hui

Copyright ( $\odot 2012$ H. L. Sneha et al. This is an open access article distributed under the Creative Commons Attribution License, which permits unrestricted use, distribution, and reproduction in any medium, provided the original work is properly cited.

The estimation of RCS of a phased array depends on various parameters, namely, array geometry, operational frequency, feed network, mutual coupling between the antenna elements and so fourth. This paper presents the estimation of RCS of linear dipole array with series-feed network by tracing the signal path from the antenna aperture into the feed network. The effect of mutual coupling exhibited by the dipole antenna is considered for three configurations namely, side by side, collinear, and parallel in echelon. It is shown that the mutual coupling affects the antenna pattern (and hence RCS) significantly for larger scan angles. Further it is inferred that the RCS of phased array can be optimized by (i) reducing the length of the dipole, (ii) termination of the isolation port of the coupler with a suitable load, and (iii) using suitable amplitude distribution.

\section{Introduction}

Radar Cross Section (RCS) of an aerospace vehicle significantly depends on the scattering of the signals by the antenna array mounted on it [1]. This antenna mode scattering is dependent on (i) geometry of the phased array, (ii) nature of the feed network employed to excite it, and (iii) mutual coupling effect. In general a phased array requires a feed network in order to function as an effective receiver or transmitter. This feed network comprises of radiating elements, phase shifters, couplers, and the terminating resistors. Depending on their mode of arrangement, the feed network may be categorized as series feed, corporate feed and space feed $[2,3]$. In order to design a series-fed phased array with specific desired characteristics several factors like the number of elements, their Interelement spacing, and beam scan angle are to be considered. Further if the spacing between the elements is less than half-wavelength, the effects of mutual coupling become predominant. These parameters of the array design are found to affect the RCS of the target considerably [4].

Mutual coupling has its influence on the impedance of the array elements, reflection coefficients, radiation pattern, and thus the RCS of phased array [5]. Every element in the antenna array will be of a definite physical size and shape. The signal incident on each of these elements will be reradiated due to various reasons like manufacturing defects, mismatches in the network, and so forth. The fields radiated from one antenna element interact with the field of the surrounding antennas which give rise to coupling. These reradiated fields when scattered along different directions affect the RCS of the target significantly. Further the effect of mutual coupling on the array performance is influenced by (i) feed network, (ii) array scan angle, and (iii) geometry of the antenna array, namely, side-by-side, collinear, and parallel-in-echelon configuration [5].

The literature available in the open domain presents several methods to estimate the antenna RCS. These include finite difference time domain (FDTD) method [6], shooting and bouncing ray (SBR) approach [7, 8], physical optics (PO) based model [9], scattering matrix approach [10], method of moments [11, 12], analytical formulation approach [13], and method of moments-particle swarm optimization (MoM-PSO) method [14].

Over the years, efforts have been put to reduce or compensate the mutual coupling effect [15]. Gupta and Ksienski [16] presented an open-circuit voltage approach for 
calculating mutual coupling in transmitting and receiving antenna array. Another approach based on the compensation of receiving mutual impedance was proposed, especially for small and compact receiving monopole array [17, 18]. Lee and Chu [19] studied the mutual coupling effect for a finite nonlinearly loaded antenna array using power series expansion technique. However, the effect of the feed network was neglected. Lu et al. [20] proposed a method to estimate the RCS of antenna array as a product of array factor and element factor, without inclusion of mutual coupling effect. Jenn and Lee [21] estimated the RCS of a series-fed phased array by considering the feed components, but ignoring the mutual coupling effect. Lee and Chu [22] included the mutual coupling factor in RCS estimation of a parallel-fed phased array using iterative scattering matrix approach. The edge effects and multiple reflections within the feed network were included. Najib et al. [23] studied the effect of mutual coupling in a phased array with butler-feed network.

In this paper, the method of estimating the RCS of phased array including mutual coupling effect and the feed network is presented. A step-by-step approach is followed to trace the signal path from the antenna aperture to the feed network, and hence obtaining the RCS of phased array including mutual coupling factor. This involves the derivation of the coupling coefficients in terms of impedance matrix. The parameters like length and radius of the dipole, antenna impedance are included. This work further presents a detailed formulation for the scattered field at each stage of the feed network. The simulation results are presented for side-by-side, collinear, and parallel-in-echelon configuration of linear dipole array. The RCS pattern obtained in the presence of mutual coupling is compared with no mutual coupling case. The authenticity of the results is established by comparing them with the trends, inferences, and results reported in the open domain.

\section{Formulation for RCS of Series-Fed Phased Antenna Array}

The RCS of an object for a plane wave incidence can be expressed as the ratio of the scattered field to the incident field. The mathematical expression for the antenna modescattered field is given by [5]

$$
\vec{E}_{n}^{s}(\theta, \phi)=\left[\frac{j \eta}{4 \lambda Z_{a}} \vec{h}\left\{\vec{h} \cdot \vec{E}^{i}(\theta, \phi)\right\} \frac{e^{-j \vec{k} \vec{R}}}{R}\right] \Gamma_{n}^{r}(\theta, \phi),
$$

where $\vec{E}_{n}^{s}$ is the scattered field of the $n$th element, $\vec{E}^{i}$ is the incident field, $(\theta, \phi)$ is direction of the signal, $Z_{a}=R_{a}+j X_{a}$ is the radiation impedance with $R_{a}=R_{r}+R_{d}, R_{a}$ is the antenna resistance, $X_{a}$ is the antenna reactance, $R_{r}$ is the radiation resistance, $R_{d}$ is the loss resistance of antenna (including conduction and dielectric losses), $\lambda$ is the wavelength, $\eta$ is the impedance of medium surrounding the antenna, $k=2 \pi / \lambda$ is the free space wave number, $R$ is the distance between the target and the observation point, $\Gamma_{n}^{r}$ is the total reflected signal returning to aperture element $n$, and $\vec{h}$ is the effective height of the antenna element.
For a lossless antenna placed in free space, $\eta=\eta_{o}=120 \pi$ ohms with $R_{d}=0$. Thus $R_{a}=R_{r}$.

The expression (1) can be thus rewritten as

$$
\vec{E}_{n}^{s}(\theta, \phi)=\left[\frac{j \eta_{o}}{4 \lambda Z_{a}} \vec{h}\left\{\vec{h} \cdot \vec{E}^{i}(\theta, \phi)\right\} \frac{e^{-j \vec{k} \vec{R}}}{R}\right] \Gamma_{n}^{r}(\theta, \phi) .
$$

For a unit amplitude incident plane wave [24], we have

$$
\vec{E}^{i}(\theta, \phi)=e^{-j \vec{k} \cdot \vec{d}_{n}} \hat{\theta}
$$

where $\vec{d}_{n}=\hat{x}(n-1) d$ represents the distance vector, with interelement spacing, $d$. The wave vector $\vec{k}$ can be expressed in terms of direction cosines and the unit vectors [25] as

$$
\vec{k}=k(\hat{x} \sin \theta \cos \phi+\hat{y} \sin \theta \sin \phi+\hat{z} \cos \theta) .
$$

The dot product in the phase factor of the incident signal can thus be simplified as

$$
\begin{gathered}
\vec{k} \cdot \vec{d}_{n}=k(\hat{x} \sin \theta \cos \phi+\hat{y} \sin \theta \sin \phi+\hat{z} \cos \theta) \hat{x}(n-1) d \\
=(n-1) k d \sin \theta \cos \phi, \\
\because \hat{x} \cdot \hat{x}=1, \quad \hat{x} \cdot \hat{y}=\hat{x} \cdot \hat{z}=0, \\
\vec{k} \cdot \vec{d}_{n}=(n-1) \alpha,
\end{gathered}
$$

where $\alpha=k d \sin \theta \cos \phi$ is the Interelement space delay of incident wave along $x$ direction.

For a closed body, with the coordinate system's origin located within it, the scalar or dot product of the outward normal and the incident wave propagation vector must be negative [2]. Mathematically, we have

$$
\vec{k} \cdot \vec{d}_{n}<0 .
$$

Hence from (3) and (5), the term

$$
e^{-j \vec{k} \cdot \vec{d}_{n}}=e^{j(n-1) \alpha} .
$$

For an $x$-polarized antenna, substitution of (7) into (2) yields

$$
\begin{aligned}
\vec{E}_{n}^{s}(\theta, \phi) & =\left[\frac{j \eta_{0}}{4 \lambda Z_{a}} h \hat{x}\left\{h \hat{x} \cdot e^{j(n-1) \alpha} \hat{\theta}\right\} \frac{e^{-j \vec{k} \vec{R}}}{R}\right] \Gamma_{n}^{r}(\theta, \phi) \\
& =\left[\frac{j \eta_{0}}{4 \lambda Z_{a}} h^{2} \cos \theta \vec{E}_{n}^{r}(\theta, \phi)\right] \frac{e^{-j \vec{k} \vec{R}}}{R} \hat{x} .
\end{aligned}
$$

This is the scattered field due to a single dipole element in the array. The overall scattered field is obtained by summing (8) over all the array elements. Consider

$$
\begin{aligned}
\vec{E}^{s}(\theta, \phi) & =\sum_{n=1}^{N} \vec{E}_{n}^{s}(\theta, \phi) \\
& =\sum_{n=1}^{N}\left[\left\{\frac{j \eta_{o}}{4 \lambda Z_{a}} h^{2}(\cos \theta) \vec{E}_{n}^{r}(\theta, \phi)\right\} \frac{e^{-j \vec{k} \vec{R}}}{R} \hat{x}\right] .
\end{aligned}
$$


This yields the overall RCS of the dipole array for a unit magnitude incident plane wave. We have

$$
\sigma(\theta, \phi)=4 \pi\left|\sum_{n=1}^{N}\left\{\frac{j \eta_{0}}{4 \lambda Z_{a}} h^{2}(\cos \theta) \vec{E}_{n}^{r}(\theta, \phi)\right\}\right|^{2} .
$$

The effective height of an $x$-polarized antenna element is given by [2]

$$
\vec{h}=h \hat{x}=\left(\frac{1}{I(0)} \int_{\Delta l} I(l) d l\right) \hat{x},
$$

where $I(0)$ is the current at the feed terminal of the dipole element, and $l$ is the dipole length.

Assuming the current distribution on the single dipole antenna to be cosine distribution, that is, $I(l)=I(0) \cos (k l)$ [5], the expression for the effective height becomes

$$
\begin{aligned}
\vec{h} & =\left(\frac{1}{I(0)} \int_{\Delta l} I(0) \cos (k l) d l\right) \hat{x} \\
& =\left(\int_{\Delta l} \cos (k l) d l\right) \hat{x} .
\end{aligned}
$$

Substitution of (12) into (10) yields

$$
\begin{aligned}
& \sigma(\theta, \phi) \\
& =4 \pi\left|\sum_{n=1}^{N}\left\{\frac{j \eta_{o}}{4 \lambda Z_{a}}\left(\int_{\Delta l} \cos (k l) d l\right)^{2}(\cos \theta) \vec{E}_{n}^{r}(\theta, \phi)\right\}\right|^{2} \\
& =4 \pi\left|F \sum_{n=1}^{N} \vec{E}_{n}^{r}(\theta, \phi)\right|^{2}
\end{aligned}
$$

where

$$
F=\frac{j \eta_{o}}{4 \lambda Z_{a}}\left(\int_{\Delta l} \cos (k l) d l\right)^{2} \cos \theta
$$

$\vec{E}_{n}^{r}(\theta, \phi)$ represents the total scattered field returning to the aperture after being reflected from the mismatches prevailing within the feed network. This factor can be computed by traversing the path of the signal as it travels through the different components of the feed network (Figure 1). Thus consider

$$
\begin{gathered}
\sigma(\theta, \phi)=4 \pi\left\{\left|\sigma_{r}(\theta, \phi)\right|^{2}+\left|\sigma_{p}(\theta, \phi)\right|^{2}+\left|\sigma_{c}(\theta, \phi)\right|^{2}\right. \\
\left.+\left|\sigma_{s}(\theta, \phi)\right|^{2}\right\} .
\end{gathered}
$$

The total RCS of phased array is the summation of individual contribution from radiating elements, phase shifters, and coupling port of the couplers.

\section{Impedance at Different Levels of the Feed Network}

The signal from the antenna aperture enters into the feed network and then pass through its different levels before reaching the receive port. The feed network is a combination of various components, namely, radiators, phase shifters, couplers, and terminating loads. Each of these components offers finite impedance to the traveling signal (Figure 2). Thus in the feed network, a significant impedance mismatch can occur at the junction. This results in the reflection of the incoming signal depending on the value of reflection coefficient, which in turn affects the total RCS of array.

The following subsections present the calculation of the impedances at each level of the feed network.

3.1. Impedance at the Terminals of the Dipole Antenna. A dipole antenna in general will have finite impedance at its feed terminals. The impedance depends on the location of the antenna, the angle of incident signal, and the mutual coupling. In this paper, the effect of mutual coupling is analyzed for three different configurations of dipole array, namely, side-by-side, collinear, and parallel-in-echelon (Figure 3).

The antenna impedance is expressed as $Z_{a_{n}}=R_{a_{n}}+j X_{a_{n}}$. The corresponding impedance matrix can be expressed as [26]

$$
z_{a_{x, y}}=\left(\begin{array}{cccc}
z_{a_{1,1}} & z_{a_{1,2}} & \cdots & z_{a_{1, N}} \\
z_{a_{2,1}} & z_{a_{2,2}} & \cdots & z_{a_{2, N}} \\
\vdots & \vdots & \ddots & \vdots \\
z_{a_{N, 1}} & z_{a_{N, 2}} & \cdots & z_{a_{N, N}}
\end{array}\right),
$$

where $z_{a_{i, i}}$ represents the self-impedance of the $i$ th element and $z_{a_{i, j}}(i \neq j)$ is the mutual impedance between $i$ th and $j$ th element. The expressions for the self- and mutual impedance between the dipoles for different configurations can be referred to Appendix A.

This yields the total antenna impedance at the $n$th element as [5]

$$
Z_{a_{n}}=\sum_{y=1}^{N} z_{a_{x, y},} \frac{I_{y}}{I_{x}}
$$

where $I_{n}$ is the current at the feed terminals of $n$th antenna element.

3.2. Impedance at the Terminals of the Phase Shifters. The impedance at the terminals of the phase shifters depends on the nature of the phase shifters. In the present work, the phase shifters are modeled as simple lossless delay lines with characteristic impedance $Z_{0}$. The length of the delay lines connected to each of the antennas depends on the phase shift required. The length of the delay-lines, $L_{n}$ is obtained from the phase shift as [27]

$$
L_{n}=\frac{\lambda}{2 \pi}\left[(n-1) k d \sin \theta_{s} \cos \phi_{s}\right] .
$$

These delay lines are connected to the antenna terminals at one end and to the coupling port at the other end. Thus the antenna impedance, which will be the input impedance for the phase shifter, will be translated along its length as per the following formula [28]:

$$
Z_{p_{n}}=Z_{0}\left[\frac{Z_{a_{n}}+j Z_{0} \tan \left((2 \pi / \lambda) L_{n}\right)}{Z_{0}+j Z_{a_{n}} \tan \left((2 \pi / \lambda) L_{n}\right)}\right] .
$$




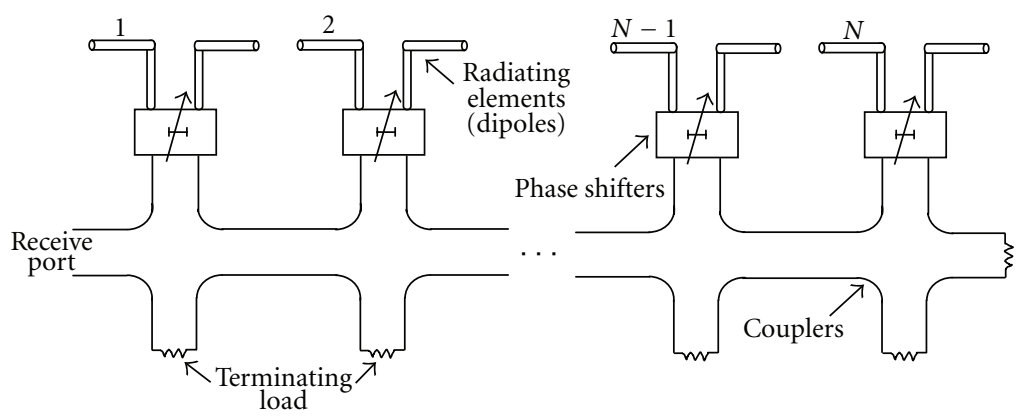

FIGURE 1: Typical series-fed network of phased array.
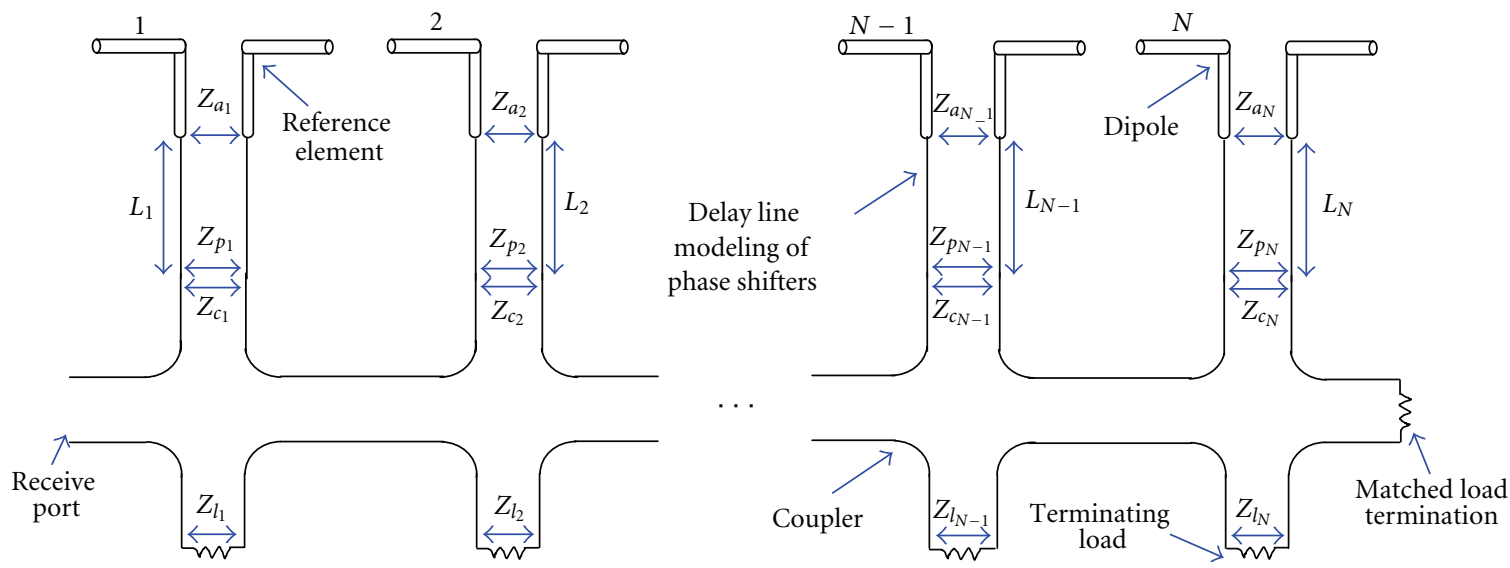

FIGURE 2: Impedances at different stages in a series-fed network.

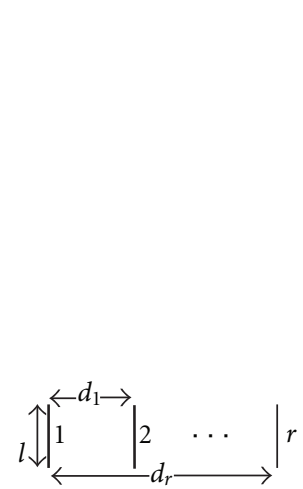

(a)

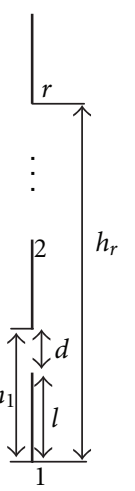

(b)

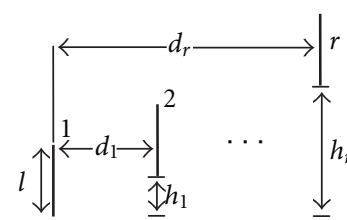

(c)
Figure 3: Schematic of dipole array. (a) Side-by-side configuration, (b) collinear configuration, and (c) parallel-in-echelon configuration.

This yields the impedance at the other end of the phase shifter, that is, $Z_{p_{n}}$ (Figure 2).

3.3. Impedance at the Coupler Terminals. The impedances at different ports of the couplers depend on type of the couplers. The feed network is assumed to have lossless fourport couplers (Figure 4).

Port 1 and Port 2 are the input and transmission ports, respectively, and form a part of the main feed line. Port 3 represents the coupling port, which connects to the antenna via phase shifter. Port 4 represents the isolated port, which is terminated with a load.

In general the terminating load is chosen as per the design, and the coupling port impedance is taken to be equal to its complex conjugate, that is, $Z_{c_{n}}=Z_{l_{n}}^{*}$. This condition ensures that there exists maximum power transfer between the coupler port and the terminating load. This in turn yields the maximum reflection coefficient at the terminating load, and thus high RCS.

\section{Scattering Contributions from Different Components of the Feed Network}

The total RCS of the target can be expressed as the sum of individual components indicating the scattering at the different levels of the feed network. The expressions for these individual contributions can be obtained by following the path of the signal as it travels from antenna aperture to the feed network. Each of these contributions can be expressed in terms of reflection and transmission coefficients of feed network elements.

4.1. RCS Component due to Scattering from Dipoles. The first source of scattering of the incident signal is the radiating element (e.g., a dipole). A well-designed antenna is expected to radiate/receive the entire energy incident on it. However 


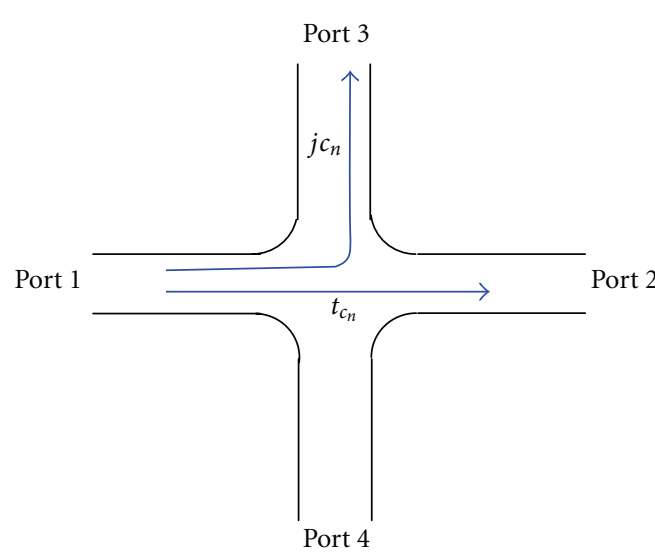

(a)

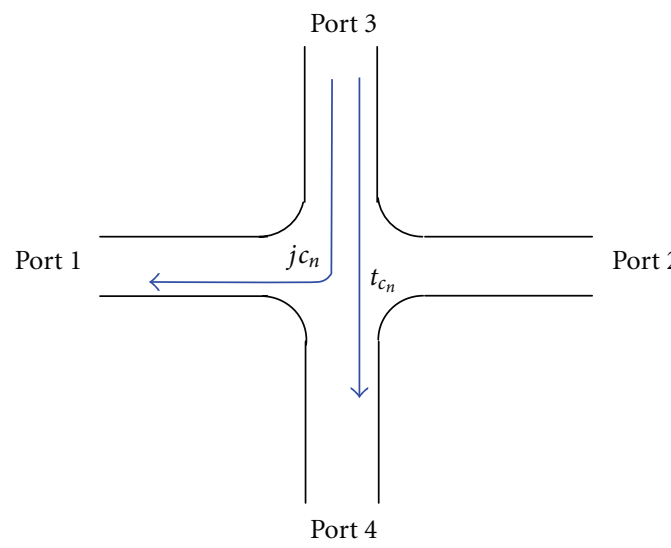

(c)

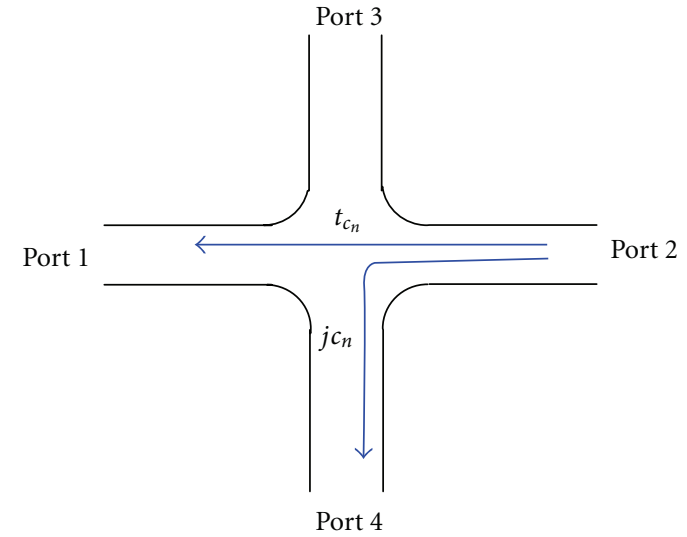

(b)

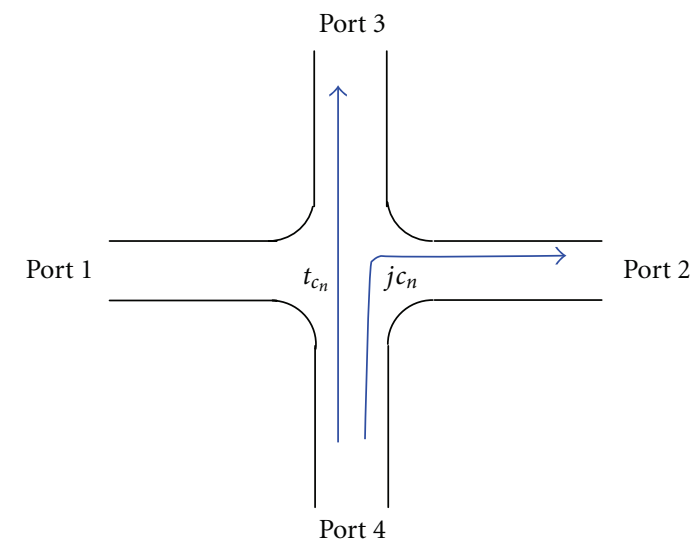

(d)

FIGURE 4: A four-port coupler showing the transmission $\left(t_{c_{n}}\right)$ and the coupling $\left(j c_{n}\right)$ coefficients.

there exists a mismatch between the antenna impedance, $Z_{a_{n}}$, and the characteristic impedance of the delay line, $Z_{0}$ connected to it. This can be modeled as the antenna connected to a load representing the remaining part of the feed network (Figure 5). Further the reflection of the incident signal can be expressed in terms of reflection coefficient $r_{r_{n}}$ of the $n$th dipole.

Mathematically, we have

$$
r_{r_{n}}=\left|\frac{Z_{a_{n}}-Z_{0}}{Z_{a_{n}}+Z_{0}}\right|
$$

This reradiated portion of the signal from adjacent antenna elements exhibits linear phase variation along the array. In terms of the reflection coefficient $r_{r_{n}}$ and the phase of the $n$th element, the scattered electric field from $n$th antenna element is given by

$$
\vec{E}_{r_{n}}^{r}(\theta, \phi)=e^{j(n-1) \alpha} r_{r_{n}} e^{j(n-1) \alpha}=r_{r_{n}} e^{j 2(n-1) \alpha} .
$$

Equation (21) represents the scattered field due to a single radiating element. The summation of (21) over all the elements of the array yields the total scattered field due to the dipole array. Thus the corresponding RCS component is given by

$$
\sigma_{r}(\theta, \phi)=F \sum_{n=1}^{N} \vec{E}_{r_{n}}^{r}(\theta, \phi)=F \sum_{n=1}^{N} r_{r_{n}} e^{j 2(n-1) \alpha} .
$$

4.2. RCS Component due to Scattering from the Phase Shifters. The portion of the signal received (not reflected) by the radiating element is transmitted to the next stage of feed network, that is, the input port of the phase shifter (modeled as lossless delay lines). This can be represented in terms of transmission coefficient of the radiating elements, $t_{r_{n}}$, given by $t_{r_{n}}^{2}=1-r_{r_{n}}^{2}$. The transmitted signal flows along the delay line to reach the coupling port of the coupler. At the end of the delay line a certain portion of the signal may be reflected back as the impedance at that point, $Z_{p_{n}}$, differs from the characteristic impedance of the line, $Z_{0}$. This is represented in terms of the reflection coefficient of the phase shifter, $r_{p_{n}}$, given by

$$
r_{p_{n}}=\left|\frac{Z_{p_{n}}-Z_{0}}{Z_{p_{n}}+Z_{0}}\right| .
$$

This reflected signal propagates back towards the radiating element so as to suffer reflection and transmission for the 


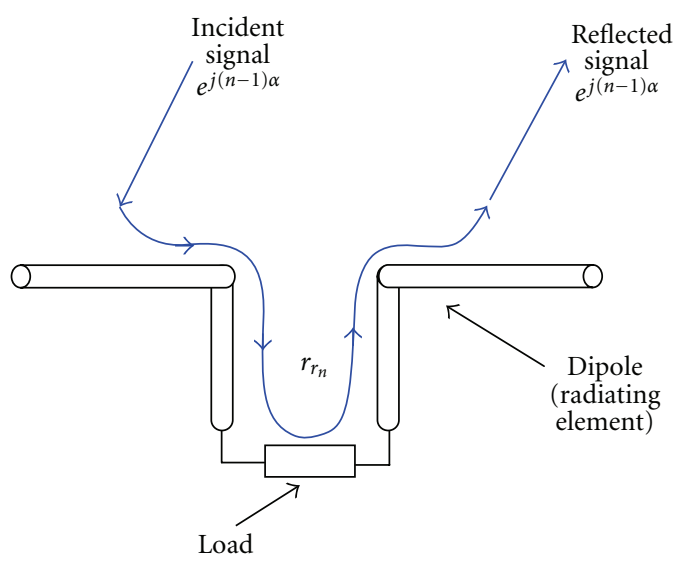

FIGURE 5: Signal reflection from the radiating element.

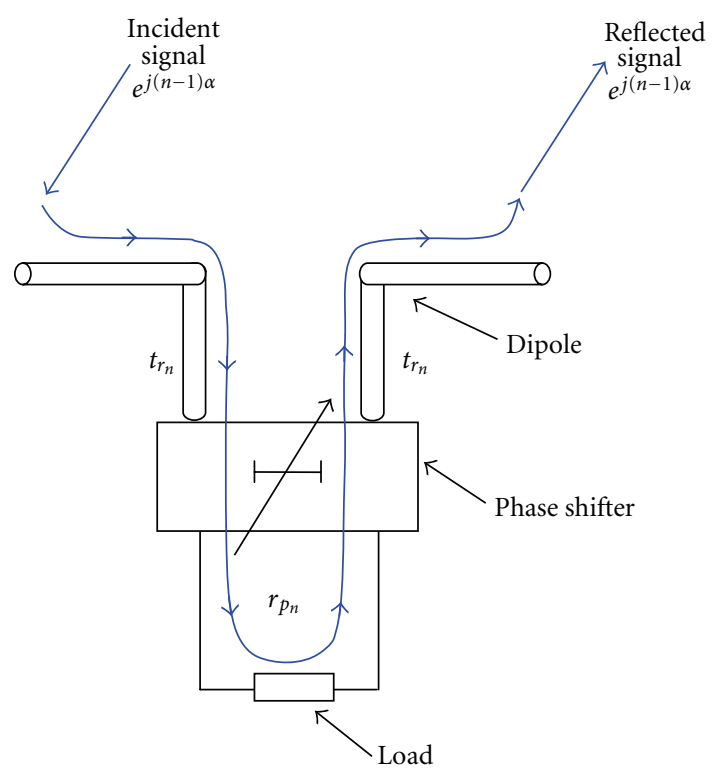

FIGURE 6: Signal reflection at the phase shifter.

second time. However, these higher-order reflections can be neglected if their magnitude is negligible. Thus following the path of the signal as shown by Figure 6, the scattered field due to $n$th phase shifter can be expressed as

$$
\begin{aligned}
\vec{E}_{p_{n}}^{r}(\theta, \phi) & =\left(e^{j(n-1) \alpha} t_{r_{n}} r_{p_{n}} t_{r_{n}} e^{j(n-1) \alpha}\right) \\
& =t_{r_{n}}^{2} r_{p_{n}} e^{j 2(n-1) \alpha} .
\end{aligned}
$$

Here the antenna is assumed to be a reciprocal device. Thus, the transmission coefficient for both the signal propagating towards the phase shifter and for the signal propagating back to the radiating element is taken as identical. This results $t_{r_{n}}^{2}$ in (24). Here the load represents the levels of feed network beyond the phase shifters. The exponential term in (24) signifies linear phase variation similar to that in the case of antenna elements.

Similar to the case of radiating elements, the summation of (24) over all the elements in phased array yields the total scattered field due to phase shifters. The corresponding RCS component is given by

$$
\sigma_{p}(\theta, \phi)=F \sum_{n=1}^{N} \vec{E}_{p_{n}}^{r}(\theta, \phi)=F \sum_{n=1}^{N} t_{r_{n}}^{2} r_{p_{n}} e^{j 2(n-1) \alpha} .
$$

4.3. RCS Component due to Scattering from the Coupling Port of the Couplers. Figure 7 shows the path of the signal, which passes through the phase shifters towards the coupling port of the coupler. This transmitted signal is determined by the transmission coefficient of the phase shifter, $t_{p_{n}}^{2}=$ $1-r_{p_{n}}^{2}$. At this junction, significant reflection can occur if the impedance at the end of the phase shifter, $Z_{p_{n}}$, and the impedance at the coupler input port, $Z_{\mathcal{c}_{n}}$, differ. The corresponding reflection coefficient $r_{c_{n}}$ is given by

$$
r_{c_{n}}=\left|\frac{Z_{c_{n}}-Z_{p_{n}}}{Z_{c_{n}}+Z_{p_{n}}}\right| \text {. }
$$

Thus, the scattered field due to $n$th coupler is expressed as

$$
\begin{aligned}
& \vec{E}_{c_{n}}^{r}(\theta, \phi) \\
& \quad=\left(e^{j(n-1) \alpha} t_{r_{n}} t_{p_{n}} e^{j(n-1) \alpha_{s}} r_{c_{n}} t_{p_{n}} e^{j(n-1) \alpha_{s}} t_{r_{n}} e^{j(n-1) \alpha}\right) \\
& \quad=\left(t_{r_{n}}^{2} t_{p_{n}}^{2} r_{c_{n}} e^{j 2(n-1) \alpha_{s}} e^{j 2(n-1) \alpha}\right) \\
& \quad=t_{r_{n}}^{2} t_{p_{n}}^{2} r_{c_{n}} e^{j 2(n-1)\left(\alpha+\alpha_{s}\right)}=t_{r_{n}}^{2} t_{p_{n}}^{2} r_{c_{n}} e^{j 2(n-1) \zeta},
\end{aligned}
$$

where $\alpha_{s}$ represents the Interelement phase to scan the antenna beam along $x$ direction. As both the radiating elements and the phase shifters are considered to be reciprocal devices, the expression (27) has terms $t_{r_{n}}^{2}$ and $t_{p_{n}}^{2}$. The exponential term in (27) represents the linear phase variation. The RCS component due to the scattering of the signal at the coupling port of the couplers is given by

$$
\begin{aligned}
\sigma_{c}(\theta, \phi) & =F \sum_{n=1}^{N} \vec{E}_{c_{n}}^{r}(\theta, \phi) \\
& =F \sum_{n=1}^{N} t_{r_{n}}^{2} t_{p_{n}}^{2} r_{c_{n}} e^{j 2(n-1) \zeta} .
\end{aligned}
$$

4.4. RCS Component due to Scattering Beyond the Coupling Port of Couplers. The path of signal not reflected at the coupling port of the coupler, depends on the nature of coupler. Assuming the couplers as lossless four-port devices, the coupling coefficient can be expressed as

$$
c_{n}=\frac{Z_{a_{n}} i_{n}^{2}}{\sum_{p=1}^{N} Z_{a_{p}} i_{p}^{2}-\sum_{q=1}^{n-1} Z_{a_{q}} i_{q}^{2}} .
$$

The detailed derivation of the coupling coefficient of coupler is given in Appendix B. The transmission coefficient of the coupler will be $t_{c_{n}}^{2}=1-c_{n}^{2}$.

The signal incident at each of the antenna elements is expected to move towards the receiving port only. However it has the ability to move towards (i) Next antenna element 


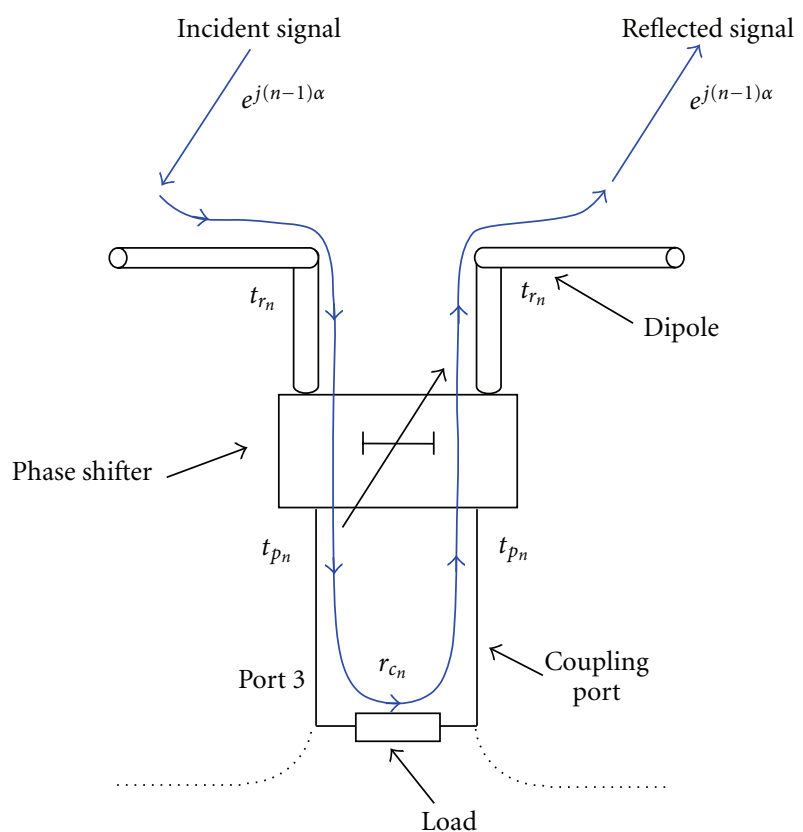

Port 1

Port 2

Port 4

Figure 7: Signal reflection at the coupling port of the coupler.

in the array, (ii) Previous antenna element in the array, (iii) Load terminating its own coupler, and (iv) Port (backward direction) through which it was received. Each of these signals gives rise to scattering and hence contributes to the RCS significantly.

The scattered field at the $n$th element of the array can be due to the signals which would be incident on it from the next $(N-n)$ elements of the array. The path of such a signal is shown in Figure 8.

Here the reflection coefficient of the terminating load, $r_{l_{n}}$ can be expressed as

$$
r_{l_{n}}=\left|\frac{Z_{l_{n}}-Z_{c_{n}}}{Z_{l_{n}}+Z_{c_{n}}}\right| .
$$

Following the path of the signals through each of the elements, the scattered electric field is given by

$$
\begin{aligned}
& \vec{E}_{1_{n}}^{r}(\theta, \phi) \\
& =\left[\left(e^{j(N-1) \alpha} t_{r_{N}} t_{p_{N}} e^{j(N-1) \alpha_{s}} j c_{N} e^{j \psi} t_{c_{N-1}} e^{j \psi} t_{c_{N-2}}\right.\right. \\
& \left.\cdots e^{j \psi} j c_{n} r_{l_{n}} t_{c_{n}} t_{p_{n}} e^{j(n-1) \alpha_{s}} t_{r_{n}} e^{j(n-1) \alpha}\right) \\
& +\left(e^{j(N-2) \alpha} t_{r_{N-1}} t_{p_{N-1}} e^{j(N-2) \alpha_{s}} j c_{N-1} e^{j \psi} t_{c_{N-2}}\right. \\
& \left.\quad \cdots e^{j \psi} j c_{n} r_{l_{n}} t_{c_{n}} t_{p_{n}} e^{j(n-1) \alpha_{s}} t_{r_{n}} e^{j(n-1) \alpha}\right) \\
& +\cdots+\left(e^{j(n) \alpha} t_{r_{n+1}} t_{p_{n+1}} e^{j(n) \alpha_{s}} j c_{n+1} e^{j \psi}\right. \\
& \left.\left.\times j c_{n} r_{l_{n}} t_{c_{n}} t_{p_{n}} e^{j(n-1) \alpha_{s}} t_{r_{n}} e^{j(n-1) \alpha}\right)\right] .
\end{aligned}
$$

Regrouping of like factors yields

$$
\begin{aligned}
& E_{1_{n}}^{r}(\theta, \phi)=\left[\left(t_{r_{N}} t_{r_{n}} t_{p_{N}} t_{p_{n}}\right)\left(j c_{N} j c_{n}\right) r_{l_{n}}\left(e^{j(N-1) \zeta} e^{j(n-1) \zeta}\right)\right. \\
& \times\left(t_{c_{N-1}} e^{j \psi} t_{c_{N-2}} e^{j \psi} \cdots t_{c_{n}} e^{j \psi}\right) \\
& +\left(t_{r_{N-1}} t_{r_{n}} t_{p_{N-1}} t_{p_{n}}\right)\left(j c_{N-1} j c_{n}\right) r_{l_{n}} \\
& \times\left(e^{j(N-2) \zeta} e^{j(n-1) \zeta}\right)\left(t_{c_{N-2}} e^{j \psi} \cdots t_{c_{n}} e^{j \psi}\right) \\
& +\cdots+\left(t_{r_{n+1}} t_{r_{n}} t_{p_{n}} t_{p_{n+1}}\right)\left(j c_{n+1} j c_{n}\right) r_{l_{n}} \\
& \left.\times\left(e^{j(n) \zeta} e^{j(n-1) \zeta}\right)\left(t_{c_{n}} e^{j \psi}\right)\right] \\
& =\left[\left(t_{r_{N}} t_{r_{n}} t_{p_{N}} t_{p_{n}}\right)\left(j c_{N} j c_{n}\right) r_{l_{n}}\left(e^{j(N-1) \zeta} e^{j(n-1) \zeta}\right)\right. \\
& \times \prod_{i=n}^{N-1} t_{c_{i}} e^{j \psi}+\left(t_{r_{N-1}} t_{r_{n}} t_{p_{N-1}} t_{p_{n}}\right)\left(j c_{N-1} j c_{n}\right) r_{l_{n}} \\
& \times\left(e^{j(N-2) \zeta} e^{j(n-1) \zeta}\right) \prod_{i=n}^{N-2} t_{c_{i}} e^{j \psi} \\
& +\cdots+\left(t_{r_{n+1}} t_{r_{n}} t_{p_{n}} t_{p_{n+1}}\right)\left(j c_{n+1} j c_{n}\right) r_{l_{n}} \\
& \left.\times\left(e^{j(n) \zeta} e^{j(n-1) \zeta}\right) \prod_{i=n}^{n} t_{c_{i}} e^{j \psi}\right] .
\end{aligned}
$$

Removing $\left(t_{r_{n}} t_{p_{n}} r_{l_{n}} j c_{n} e^{j(n-1) \zeta}\right)$ as common yields

$$
\begin{aligned}
& \vec{E}_{1_{n}}^{r}(\theta, \phi)=\left[t_{r_{n}} t_{p_{n}} r_{l_{n}} j c_{n} e^{j(n-1) \zeta}\right. \\
& \times\left\{\left(t_{r_{N}} t_{p_{N}} j c_{N} e^{j(N-1) \zeta}\right) \prod_{i=n}^{N-1} t_{c_{i}} e^{j \psi}\right. \\
& +\left(t_{r_{N-1}} t_{p_{N-1}} j c_{N-1} e^{j(N-2) \zeta}\right) \prod_{i=n}^{N-2} t_{c_{i}} e^{j \psi} \\
& \left.\left.+\cdots+\left(t_{r_{n+1}} t_{p_{n+1}} j c_{n+1} e^{j(n) \zeta}\right) \prod_{i=n}^{n} t_{c_{i}} e^{j \psi}\right\}\right], \\
& \vec{E}_{1_{n}}^{r}(\theta, \phi)=\left[t_{r_{n}} t_{p_{n}} r_{l_{n}} j c_{n} e^{j(n-1) \zeta}\right. \\
& \left.\times \sum_{m=n+1}^{N}\left(t_{r_{m}} t_{p_{m}} j c_{m} e^{j(m-1) \zeta} \prod_{i=n}^{m-1} t_{c_{i}} e^{j \psi}\right)\right] .
\end{aligned}
$$




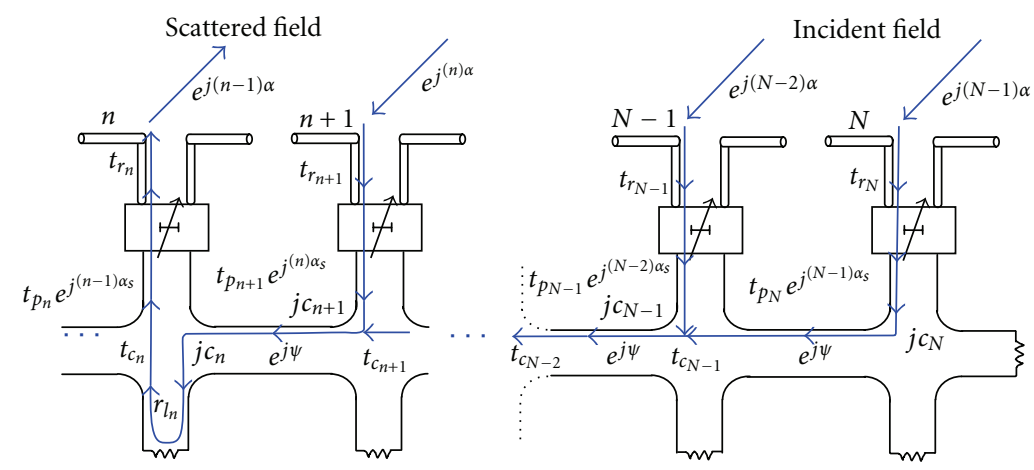

Figure 8: Path of the signal traveling towards the $n$th element from the next $(N-n)$ elements.

The scattered field at the same element $n$ may also be affected by the signals, which travel from the previous $n-1$ elements of the array towards it. The path of such a signal is shown in Figure 9.

Following the path of the signals through each of the antenna elements, one gets

$$
\begin{aligned}
& \vec{E}_{2_{n}}^{r}(\theta, \phi) \\
& =\left[\left(e^{j 0 \alpha} t_{r_{1}} t_{p_{1}} e^{j 0 \alpha_{s}} t_{c_{1}} r_{l_{1}} j c_{1} e^{j \psi} t_{c_{2}} e^{j \psi}\right.\right. \\
& \left.\quad \cdots t_{c_{n-1}} e^{j \psi} j c_{n} t_{p_{n}} e^{j(n-1) \alpha_{s}} t_{r_{n}} e^{j(n-1) \alpha}\right) \\
& +\left(e^{j 1 \alpha} t_{r_{2}} t_{p_{2}} e^{j 1 \alpha_{s}} t_{c_{2}} r_{l_{2}} j c_{2} e^{j \psi} t_{c_{3}} e^{j \psi}\right. \\
& \left.\quad \cdots t_{c_{n-1}} e^{j \psi} j c_{n} t_{p_{n}} e^{j(n-1) \alpha_{s}} t_{r_{n}} e^{j(n-1) \alpha}\right) \\
& +\cdots+\left(e^{j(n-2) \alpha} t_{r_{n-1}} t_{p_{n-1}} e^{j(n-2) \alpha_{s}} t_{c_{n-1}}\right. \\
& \left.\left.\quad \times r_{l_{n-1}} j c_{n-1} e^{j \psi} j c_{n} t_{p_{n}} e^{j(n-1) \alpha_{s}} t_{r_{n}} e^{j(n-1) \alpha}\right)\right] .
\end{aligned}
$$

Regrouping of like factors yields

$$
\begin{aligned}
& \vec{E}_{2_{n}}^{r}(\theta, \phi) \\
& =\left[\left(t_{r_{n}} t_{r_{1}} t_{p_{n}} t_{p_{1}}\right)\left(j c_{1} j c_{n}\right) r_{l_{1}}\left(e^{j 0 \zeta} e^{j(n-1) \zeta}\right)\right. \\
& \times\left(t_{c_{1}} e^{j \psi} t_{c_{2}} e^{j \psi} \cdots t_{c_{n-1}} e^{j \psi}\right) \\
& +\left(t_{r_{n}} t_{r_{2}} t_{p_{n}} t_{p_{2}}\right)\left(j c_{2} j c_{n}\right) r_{l_{2}}\left(e^{j 1 \zeta} e^{j(n-1) \zeta}\right) \\
& \times\left(t_{c_{2}} e^{j \psi} \cdots t_{c_{n-1}} e^{j \psi}\right) \\
& +\cdots+\left(t_{r_{n}} t_{r_{n-1}} t_{p_{n}} t_{p_{n-1}}\right)\left(j c_{n-1} j c_{n}\right) r_{l_{n-1}} \\
& \left.\times\left(e^{j(n-2) \zeta} e^{j(n-1) \zeta}\right)\left(t_{c_{n-1}} e^{j \psi}\right)\right] \\
& =\left[\left(t_{r_{n}} t_{r_{1}} t_{p_{n}} t_{p_{1}}\right)\left(j c_{1} j c_{n}\right) r_{l_{1}}\left(e^{j 0 \zeta} e^{j(n-1) \zeta}\right) \prod_{i=1}^{n-1} t_{c_{i}} e^{j \psi}\right. \\
& +\left(t_{r_{n}} t_{r_{2}} t_{p_{n}} t_{p_{2}}\right)\left(j c_{2} j c_{n}\right) r_{l_{2}}\left(e^{j 1 \zeta} e^{j(n-1) \zeta}\right) \prod_{i=2}^{n-1} t_{c_{i}} e^{j \psi} \\
& +\cdots+\left(t_{r_{n}} t_{r_{n-1}} t_{p_{n}} t_{p_{n-1}}\right)\left(j c_{n-1} j c_{n}\right) r_{l_{n-1}} \\
& \left.\times\left(e^{j(n-2) \zeta} e^{j(n-1) \zeta}\right) \prod_{i=n-1}^{n-1} t_{c_{i}} e^{j \psi}\right] .
\end{aligned}
$$

Removing $\left(t_{r_{n}} t_{p_{n}} j c_{n} e^{j(n-1) \zeta}\right)$ as common yields

$$
\begin{aligned}
\vec{E}_{2_{n}}^{r}(\theta, \phi) & \\
= & {\left[t_{r_{n}} t_{p_{n}} j c_{n} e^{j(n-1) \zeta}\right.} \\
& \times\left\{t_{r_{1}} t_{p_{1}} r_{l_{1}} j c_{1} e^{j 0 \zeta} \prod_{i=1}^{n-1} t_{c_{i}} e^{j \psi}\right. \\
& +t_{r_{2}} t_{p_{2}} r_{l_{2}} j c_{2} e^{j 1 \zeta} \prod_{i=2}^{n-1} t_{c_{i}} e^{j \psi}+\cdots \\
& \left.\left.+t_{r_{n-1}} t_{p_{n-1}} r_{l_{n-1}} j c_{n-1} e^{j(n-2) \zeta} \prod_{i=n-1}^{n-1} t_{c_{i}} e^{j \psi}\right\}\right]
\end{aligned}
$$

$$
=\left[t_{r_{n}} t_{p_{n}} j c_{n} e^{j(n-1) \zeta} \sum_{m=1}^{n-1} t_{r_{m}} t_{p_{m}} r_{l_{m}} j c_{m} e^{j(m-1) \zeta} \prod_{i=m}^{n-1} t_{c_{i}} e^{j \psi}\right] .
$$

The scattered field at the $n$th element can also be affected due to the signal which moves towards the load terminating its own coupler, as shown in Figure 10. This results in the selfscattering of the incident signal expressed as

$$
\begin{aligned}
\vec{E}_{3_{n}}^{r}(\theta, \phi)= & \left(e^{j(n-1) \alpha} t_{r_{n}} t_{p_{n}} e^{j(n-1) \alpha_{s}} t_{c_{n}}\right) \\
& \times r_{l_{n}}\left(t_{c_{n}} t_{p_{n}} e^{j(n-1) \alpha_{s}} t_{r_{n}} e^{j(n-1) \alpha}\right) \\
= & r_{l_{n}} t_{r_{n}}^{2} t_{p_{n}}^{2} t_{c_{n}}^{2} e^{j 2(n-1) \alpha} e^{j 2(n-1) \alpha_{s}}, \\
\vec{E}_{3_{n}}^{r} & (\theta, \phi)=r_{l_{n}} t_{r_{n}}^{2} t_{p_{n}}^{2} t_{c_{n}}^{2} e^{j 2(n-1) \zeta} .
\end{aligned}
$$

The signal, which succeeds in overcoming all the abovementioned scattering sources, moves towards the receiving port (Figure 11). However if there exists a mismatch between the receiving port and the input port of the coupler, 

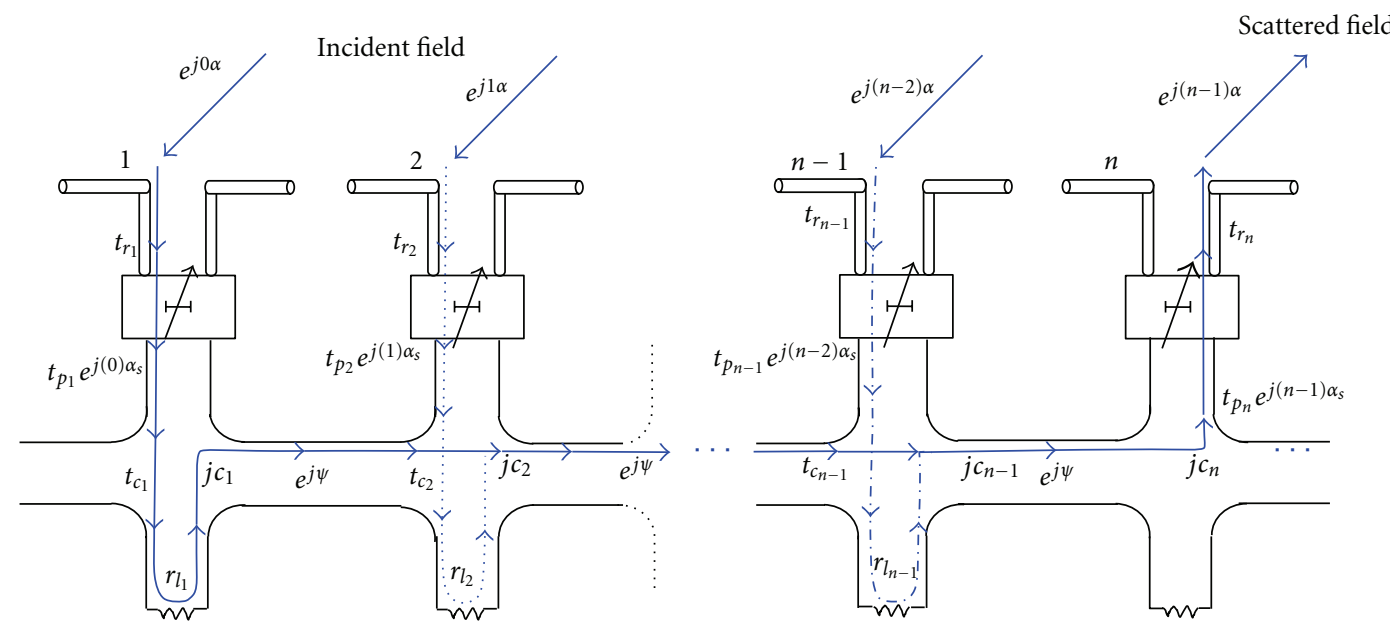

FIGURE 9: Path of the signal traveling towards the $n$th element from the previous $(n-1)$ elements.

significant scattering can occur even at this point. The reflection coefficient at this input port can be expressed as

$$
r_{i n}=\frac{Z_{12}-Z_{0}}{Z_{12}+Z_{0}}
$$

where $Z_{12}$ is the impedance at the input port of the first coupler in the array, and $Z_{0}$ is the characteristic impedance of the coaxial cable connected to the input port of the first coupler.

The scattered field at the junction of receiving port and input port of coupler is expressed as

$$
\begin{aligned}
& \vec{E}_{4_{n}}^{r}(\theta, \phi) \\
& =\left[\left(e^{j(n-1) \alpha} t_{r_{n}} t_{p_{n}} e^{j(n-1) \alpha_{s}} j c_{n} e^{j \psi} t_{c_{n-1}} \cdots e^{j \psi} t_{c_{2}} e^{j \psi} t_{c_{1}}\right) r_{i n}\right. \\
& \left.\quad \times\left(t_{c_{1}} e^{j \psi} t_{c_{2}} e^{j \psi} \cdots t_{c_{n-1}} e^{j \psi} j c_{n} t_{p_{n}} e^{j(n-1) \alpha_{s}} t_{r_{n}} e^{j(n-1) \alpha}\right)\right] .
\end{aligned}
$$

Rearrangement of like factors yields

$$
\begin{aligned}
\vec{E}_{4_{n}}^{r}(\theta, \phi)= & {\left[t_{r_{n}}^{2} t_{p_{n}}^{2} e^{j 2(n-1) \alpha} e^{j 2(n-1) \alpha_{s}}\left(j c_{n}\right)^{2}\right.} \\
& \left.\times r_{i n}\left(t_{c_{1}} e^{j \psi} t_{c_{2}} e^{j \psi} \cdots t_{c_{n-1}} e^{j \psi}\right)^{2}\right], \\
\vec{E}_{4_{n}}^{r}(\theta, \phi)= & {\left[r_{i n} t_{r_{n}}^{2} t_{p_{n}}^{2}\left(j c_{n}\right)^{2} e^{j 2(n-1) \zeta}\left(\prod_{i=1}^{n-1} t_{c_{i}} e^{j \psi}\right)^{2}\right] . }
\end{aligned}
$$

The total field due to the signal scattering beyond the coupling port of couplers is given by

$$
\vec{E}_{s_{n}}^{r}(\theta, \phi)=\vec{E}_{1_{n}}^{r}(\theta, \phi)+\vec{E}_{2_{n}}^{r}(\theta, \phi)+\vec{E}_{3_{n}}^{r}(\theta, \phi)+\vec{E}_{4_{n}}^{r}(\theta, \phi) .
$$

The corresponding RCS component is expressed as

$$
\sigma_{s}(\theta, \phi)=F \sum_{n=1}^{N} \vec{E}_{s_{n}}^{r}(\theta, \phi) .
$$

Substitution of (22), (25), (28), and (42) into (15) gives the total RCS for the phased array due to the mismatches of the feed network. The normalized RCS of phased array is expressed as

$$
\begin{aligned}
\sigma(\theta, \phi)=\frac{4 \pi}{\lambda^{2}}\{ & \left|\sigma_{r}(\theta, \phi)\right|^{2}+\left|\sigma_{p}(\theta, \phi)\right|^{2} \\
& \left.+\left|\sigma_{c}(\theta, \phi)\right|^{2}+\left|\sigma_{s}(\theta, \phi)\right|^{2}\right\} .
\end{aligned}
$$

\section{Results and Discussion}

In this section, the simulation results for the RCS of a linear series-fed dipole array are presented including mutual coupling factor. The simulations have been done using the Fortran 90 software code (developed by the authors) based on the formulation described above. The graphs are plotted using the graphical subroutine Sigma plot v.10. The variation of the RCS of antenna array due to the changes in the design parameters of the array is analyzed. The RCS pattern of a linear series-fed phased array is validated against the results in open domain. In the earlier work [29], the formulation for RCS was done for a phased array of infinitesimal dipoles $(l \ll \lambda)$ fed by a series-feed network. The mutual coupling and edge effects were not taken into account. Although these assumptions simplify the RCS estimation of the array, they are unacceptable in a practical scenario. In this paper, an attempt is made to overcome some of these limitations with acceptable increase in the computational complexity.

The impedance parameter is included into the expression of coupling coefficients of the coupler in feed network. In order to validate with the earlier results (without mutual coupling), the impedance matrix of array is taken as identity matrix. This cancels the mutual coupling effect. Figure 12 shows the RCS pattern of 50-element series-fed array with and without inclusion of impedance in the coupling coefficients formulation. The parameters are chosen as $\theta_{s}=$ $0^{\circ}, \psi=\pi / 2, d=0.4 \lambda, l=0.5 \lambda$, and $r_{r}=r_{p}=r_{c}=r_{l}=$ 0.2 with uniform unit amplitude distribution. It is evident that both the RCS patterns are in excellent match. This 


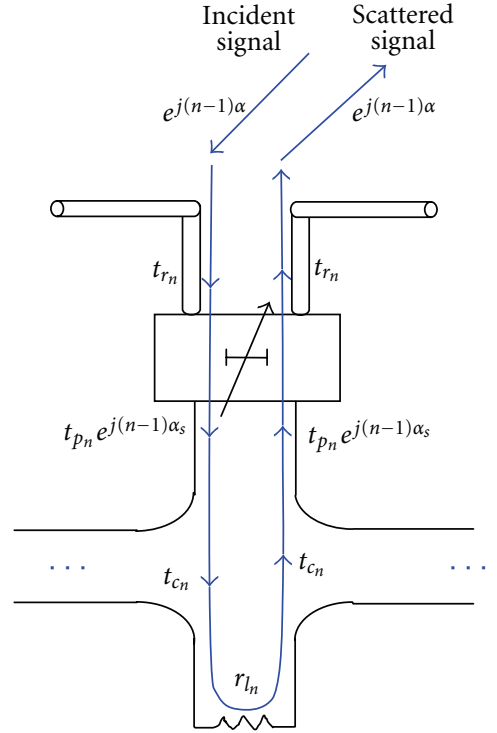

Figure 10: Path of the signal traveling towards the load terminating its own coupler.

authenticates the RCS estimation of phased array including mutual coupling factor.

Next step includes the effect of effective height of the dipole antenna element on the RCS of phased array. Although an antenna is specified using its physical length (height), its effective aperture differs from the physical one. Thus an accurate computation of the target RCS is expected to include the effective height rather than physical height of antenna element. The effective height of the antenna depends on the nature of the current distribution over its surface [2]. In the present work, the length of dipole antenna is taken as $0.003 \lambda$ in order to satisfy the assumption of infinitesimal dipole. The simulated RCS pattern (Figure 13) is compared with the earlier result using physical height of dipole [29]. The excellent match between the RCS patterns establishes the authenticity of the formulation.

Next, the RCS of phased array is calculated using antenna impedance instead of antenna resistance. This is done in order to include the finite radius of the dipole antenna element in the expression of antenna impedance, and hence RCS of phased array. In earlier work, radius of dipole antenna was not included, assuming to be infinitesimally small.

It is necessary to choose the radius and length of the dipole element appropriately. Usually the dipole is taken to be a thin wire. In the present work, the radius of the wire is fixed as $10^{-5} \lambda$. Next the antenna is assumed to be resonant. The antenna reactance is expected to vanish for a dipole of resonant length. Hence the length of dipole is taken as $0.488 \lambda$. This combination of length and radius of dipole antenna yields a radiation reactance of $-7.566 \times 10^{-5} \Omega$, which is almost equal to zero. The simulated RCS pattern is shown in Figure 14(a). The RCS results are compared with and without using radiation reactance, keeping all other parameters the same. It can be seen that the level of RCS pattern is different for this choice of dipole length and Interelement spacing. The difference in the RCS patterns can be reduced for smaller Interelement spacing, keeping dipole length constant, as shown in Figure 14(b). This observation drives attention that RCS of phased array can be controlled by multivariant optimization [30-32]. The parameters that can be optimized are the Interelement spacing $[4,13]$, dipole length, or geometry.

In Figures 12 through 14 the electric field scattered due to the termination of the receive port of the array was considered to be [21]

$$
\vec{E}_{4_{n}}^{r}(\theta, \phi)=r_{l} t_{r}^{2} t_{p}^{2}\left\{\sum_{n=1}^{N} j c_{n} e^{j(n-1) \zeta} \prod_{i=1}^{n-1} t_{c_{i}} e^{j \psi}\right\}^{2} .
$$

However if the scattered electric field is calculated by tracing the signal path, one gets

$$
\vec{E}_{4_{n}}^{r}(\theta, \phi)=\sum_{n=1}^{N}\left[r_{i n} t_{r_{n}}^{2} t_{p_{n}}^{2}\left(j c_{n}\right)^{2} e^{j 2(n-1) \zeta}\left(\prod_{i=1}^{n-1} t_{c_{i}} e^{j \psi}\right)^{2}\right] .
$$

The RCS pattern obtained using (45), that is, through tracing the signal path, is shown in Figure 15. It can be observed that the load reflection lobe is absent in the RCS pattern when compared to the RCS pattern obtained using (44). Lu et al. [20] present the RCS pattern for a series-fed $60 \times 60$ planar dipole array by considering the effect of feed network. Here the radiation resistance of dipole antenna is taken as $R_{a}=24.7(k l)^{2.4}$. Based on Lu et al. [20] formulation, the RCS pattern for a 30-element series-fed linear dipole array is shown in Figure 16. The parameters considered are $N=30$, $\theta_{s}=0^{\circ}, \psi=\pi / 2, d=0.4 \lambda, l=0.5 \lambda$, and $r_{r}=r_{p}=r_{c}=$ $r_{l}=0.2$, with uniform unit amplitude distribution. It can be observed that the RCS patterns in Figures 15 and 16 are similar.

Next, mutual coupling effect is included in the estimation of RCS of dipole array in side-by-side configuration. Figure 17 (a) presents the RCS pattern of 10-element dipole array with and without mutual coupling. Other parameters considered are $\theta_{s}=0^{\circ}, \psi=\pi / 2, d=0.1 \lambda, l=$ $0.5 \lambda, a=10^{-5} \lambda, Z_{0}=150 \Omega$, and $Z_{l}=225 \Omega$. The amplitude distribution is taken as uniform distribution with unit amplitude. Similar RCS patterns are shown in Figures 17 (b) and 17 (c) for 20- and 40-element series-fed linear dipole arrays, respectively. It is apparent that the mutual coupling effect changes the RCS pattern of the dipole array significantly.

Figure 18 presents the variation in RCS pattern of dipole array (in collinear configuration) due to beam scanning. The beam scanning includes $0^{\circ}, 45^{\circ}$, and $85^{\circ}$. The other parameters considered are $N=30, Z_{0}=75 \Omega$, and $Z_{l}=$ $150 \Omega$. It can be seen that the RCS pattern of collinear dipole array with and without mutual coupling is almost identical. However, the difference between the RCS patterns is noticeable with increase in scan angle $\theta_{s}$ from $0^{\circ}$ to $45^{\circ}$, or $85^{\circ}$. This is in accordance with the reported inference [22] that the mutual coupling affects both the array and RCS pattern significantly for large scan angles. The variation of the RCS pattern for different scan angles $\left(0^{\circ}, 45^{\circ}\right.$, and $\left.85^{\circ}\right)$ in case of a parallel-in-echelon and a side-by-side dipole array is shown by Figures 19 and 20, respectively. The parameters 


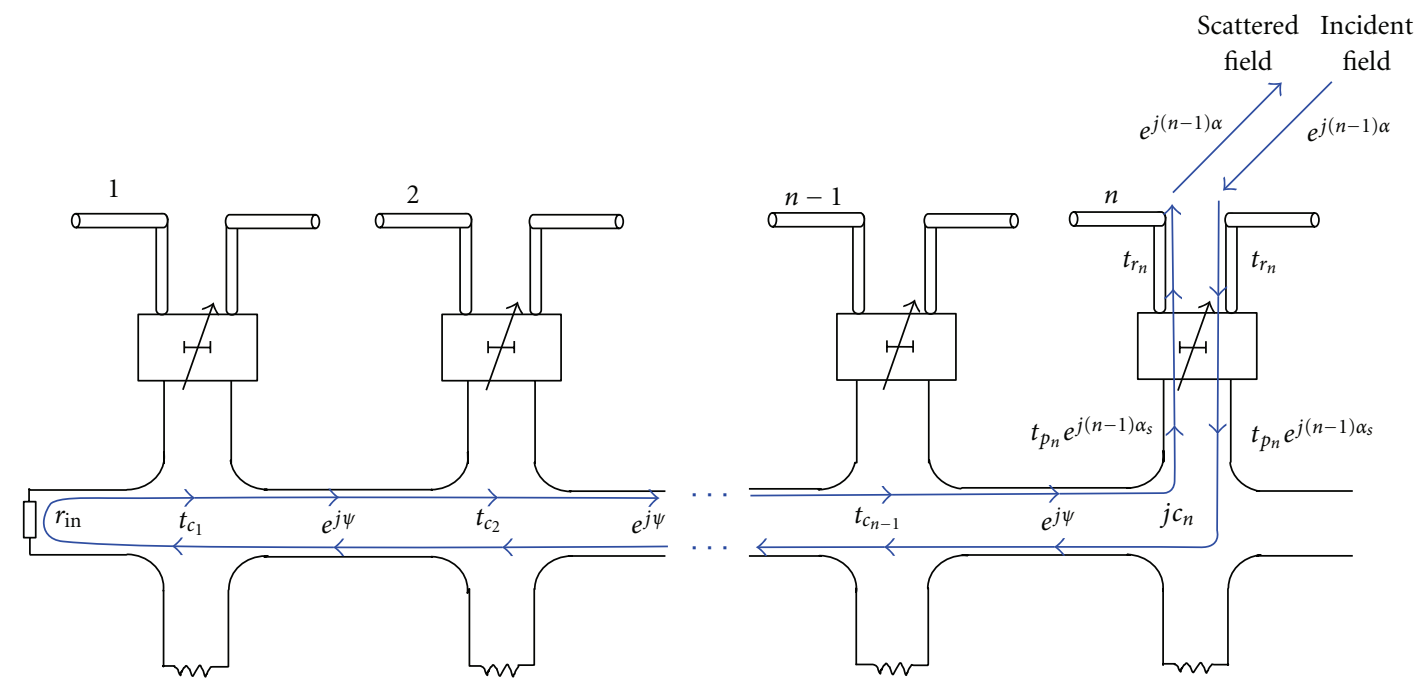

FIGURE 11: Path of the signal travelling towards the load terminating the input port.

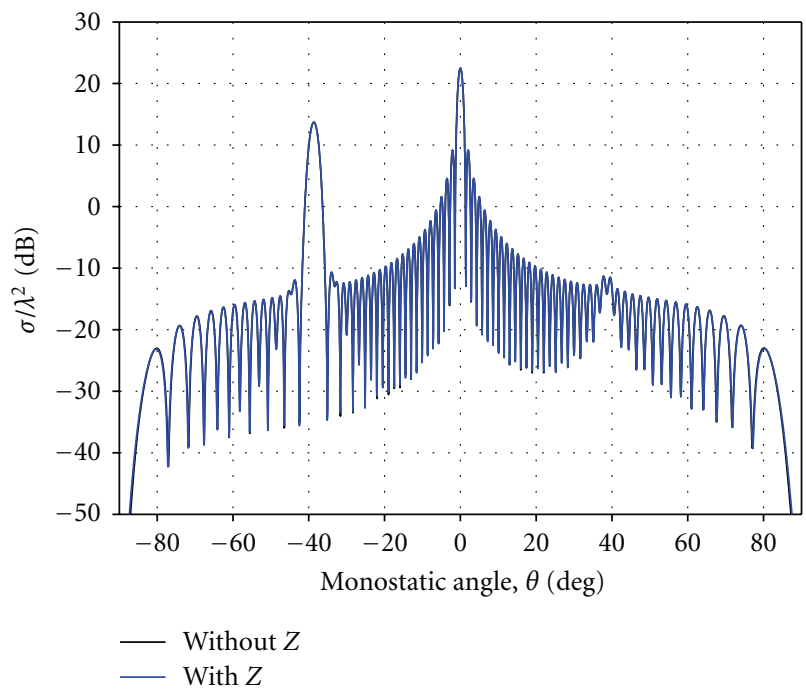

FIGURE 12: RCS pattern of a 50-element series-fed phased array. $\theta_{s}=0^{\circ}, \psi=\pi / 2, d=0.4 \lambda, l=0.5 \lambda$, and $r_{r}=r_{p}=r_{c}=r_{l}=0.2$; uniform unit amplitude distribution.

are taken to be same as in Figure 18 except for $Z_{0}$ and $Z_{l}$. The values of $Z_{0}$ and $Z_{l}$ are chosen to be $125 \Omega$, and $235 \Omega$ in case of parallel-in-echelon and $150 \Omega$ and $280 \Omega$ for side-byside configuration, respectively. It can be observed that the trend observed due to the variation of scan angle is same for all three configurations. However the difference in the RCS pattern with and without mutual coupling is least in collinear configuration of dipole array.

The estimation of RCS can provide a profitable insight for reducing the scattered field. Variation of certain design parameters of the feed network can reduce the RCS considerably. RCS of phased array can be reduced significantly by reducing the number of elements in the phased array [29]. However if the number of elements is less, the directivity of the array gets affected adversely. The other feasibility is to

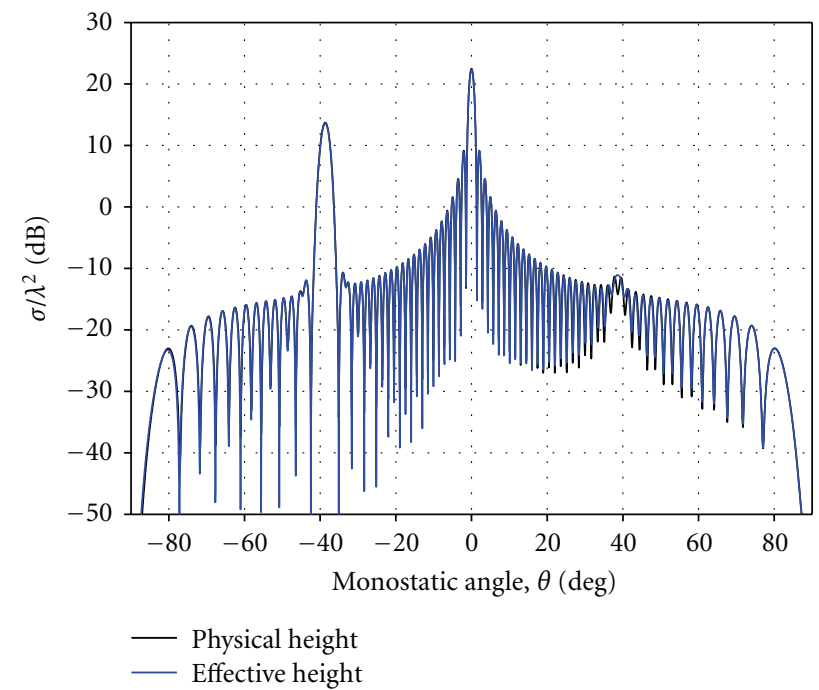

Figure 13: RCS pattern of series-fed linear array. $N=50, \theta_{s}=0^{\circ}$, $\psi=\pi / 2, d=0.4 \lambda, l=0.003 \lambda$ and $r_{r}=r_{p}=r_{c}=r_{l}=0.2$; uniform unit amplitude distribution.

reduce the antenna length, which in turn reduces the effective area of the antenna array. Figure 21(a) shows the RCS for dipoles of length $\lambda / 2$ and $\lambda / 3$ at a scan angle of $0^{\circ}$. Other parameters are taken to be $N=30, \psi=\pi / 2, d=0.1 \lambda$, $a=10^{-5} \lambda, Z_{0}=125 \Omega$, and $Z_{l}=235 \Omega$. The excitation is considered to be unit amplitude uniform distribution. The computed results are for parallel-in-echelon configuration. The plot shows that the RCS reduces as the length of the dipoles reduces. However the trend of the RCS pattern seems to be independent of the dipole length. Figure 21(b) shows the RCS pattern for dipole length variation with the same parameters but for a scan angle of $70^{\circ}$. The observations found are identical to that of Figure 21(a). This indicates that the reduction in the dipole length leads to the reduction in RCS for any scan angle. However the variation in dipole length will affect the resonant frequency of the 


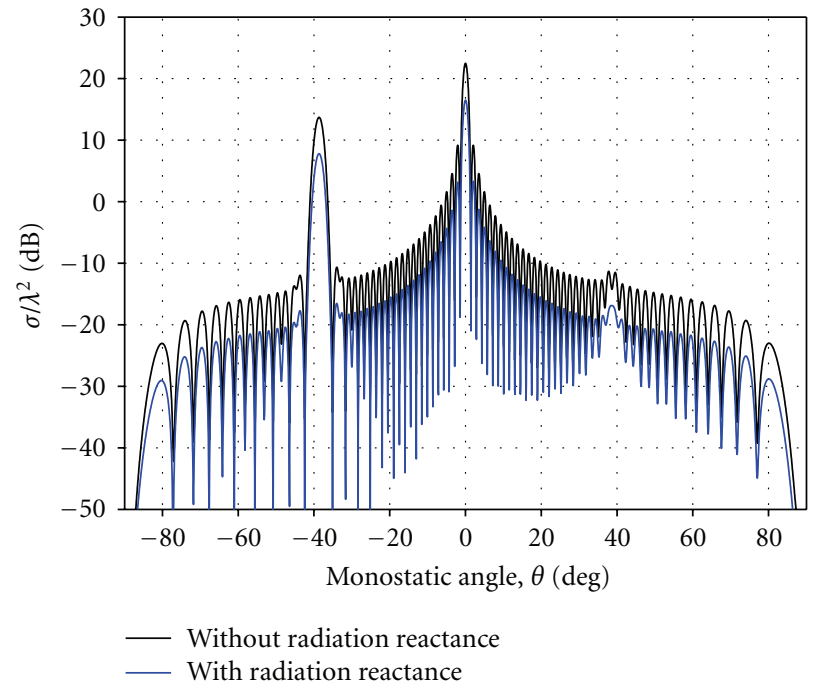

(a) $d=0.4 \lambda$

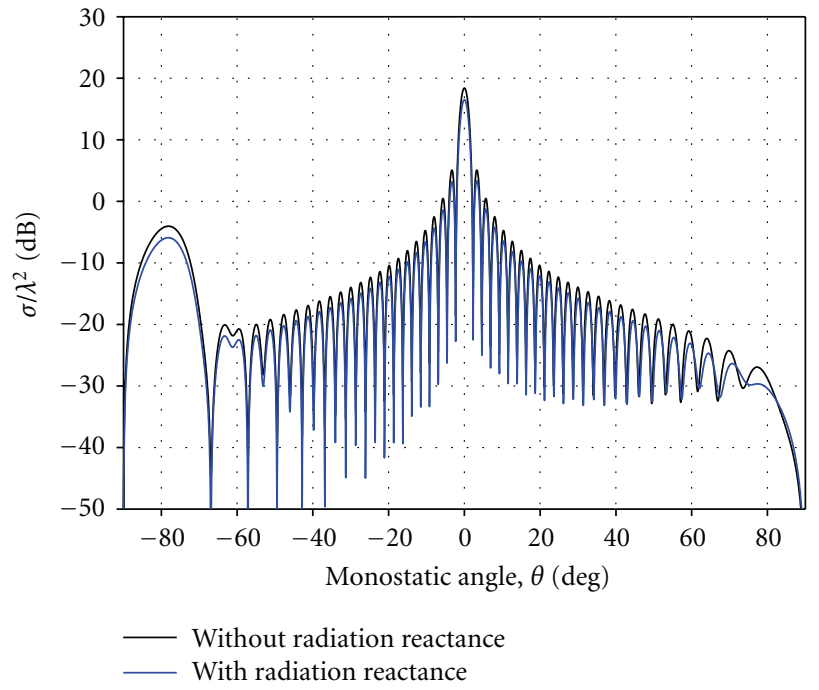

(b) $d=0.25 \lambda$

FIGURE 14: RCS of series-fed linear array. $N=50, \theta_{s}=0^{\circ}, \psi=\pi / 2, l=0.488 \lambda$ and $r_{r}=r_{p}=r_{c}=r_{l}=0.2$; uniform unit amplitude distribution.

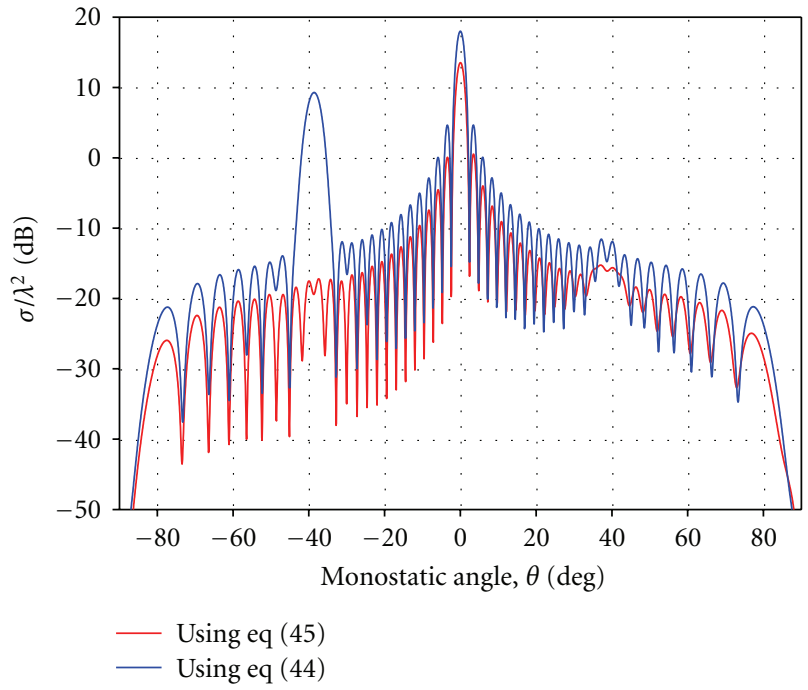

Figure 15: RCS of series-fed linear array. $N=30, \theta_{s}=0^{\circ}, \psi=\pi / 2$, $d=0.4 \lambda, l=0.5 \lambda$, and $r_{r}=r_{p}=r_{c}=r_{l}=0.2$; uniform unit amplitude distribution.

array. Therefore, reducing the RCS of a phased array just by reducing the dipole length is not recommended. Other factors like load termination or aperture distribution are also required to be chosen suitably.

Next, the dependency of the array RCS on the amplitude distribution of the current feed exciting the couplers is studied. The variations in the current at the antenna terminals affect the power distribution and hence vary the coupling coefficients of the couplers. Figure 22 shows the RCS pattern for various amplitude distributions, namely, uniform, cosine squared on a pedestal, Doplh-Chebyshev, and Taylor. The parameters chosen are of $N=30, \theta_{s}=$ $0^{\circ}, \psi=\pi / 2, d=0.1 \lambda, l=0.5 \lambda, a=10^{-5} \lambda$ for $Z_{0}=150 \Omega$,

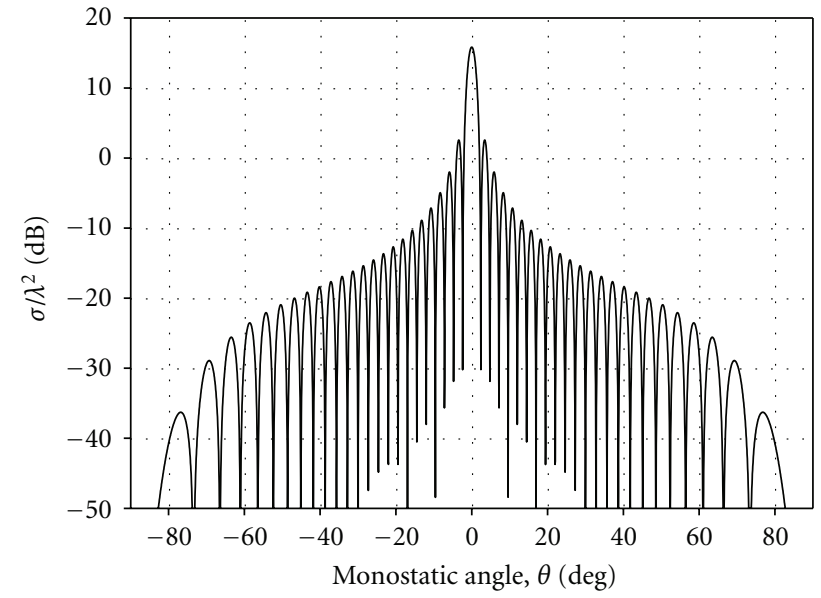

FIGURE 16: RCS of series-fed 30-element linear dipole array. $\theta_{s}=0^{\circ}$, $d=0.4 \lambda, l=0.5 \lambda$, and $r=0.2$.

and $Z_{l}=280 \Omega$. The mutual coupling factor is considered for side-by-side configuration of dipole array. It can be seen that the specular lobe has the same level irrespective of the amplitude distribution. However the levels of the side lobes vary considerably and are found to be maximum for DolphChebyshev distribution.

The termination of the isolated port of couplers varies the reflection coefficient at their respective terminal. This varies the amount of scattered field and hence the RCS of phased array. Figure 23 shows the RCS pattern for terminating impedances of $Z_{l}=0 \Omega, 50 \Omega$, and $280 \Omega$. Other parameters are kept to be the same as in the previous case (Figure 22). It can be observed that the RCS pattern for the termination of $280 \Omega$ is lower than that for short circuit or for a termination of $50 \Omega$. However the trend of the RCS pattern remains almost independent of the terminating load value. The result 


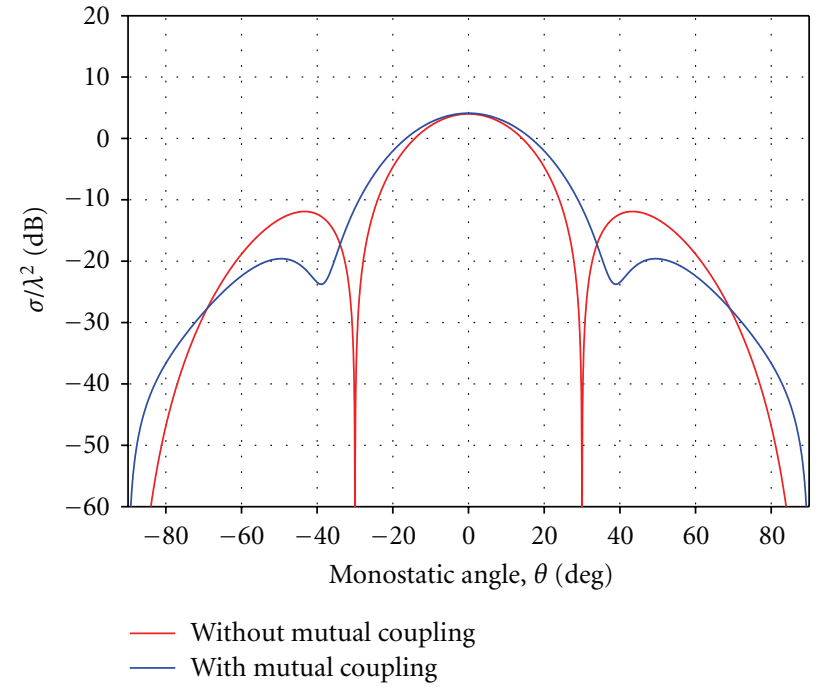

(a) $N=10, Z_{l}=225 \Omega$

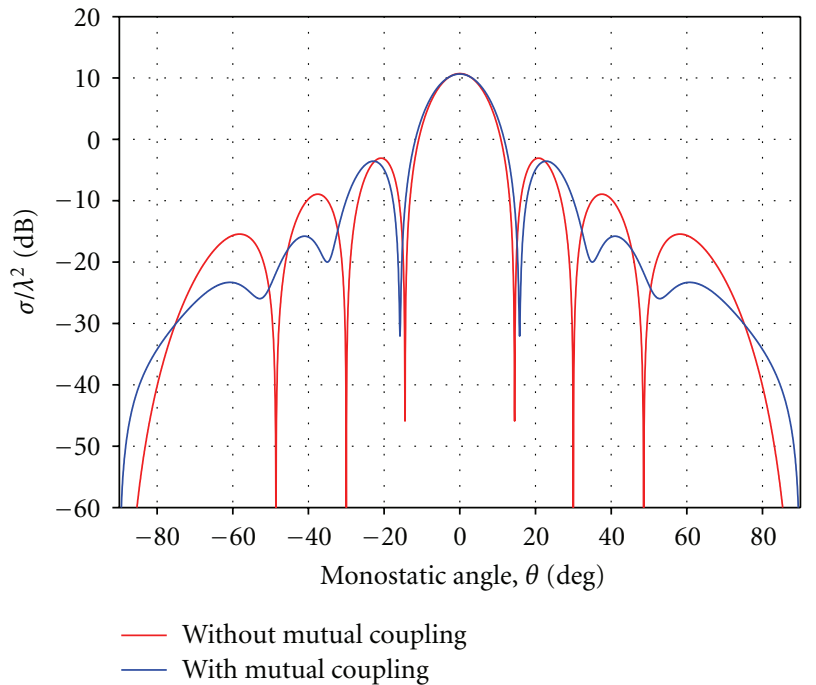

(b) $N=20, Z l=270 \Omega$

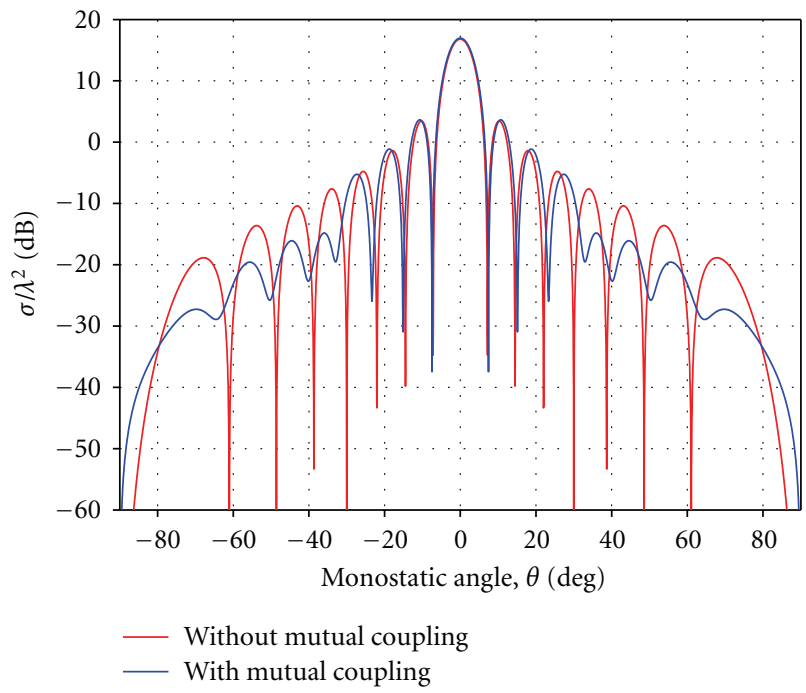

(c) $N=40, Z_{l}=275 \Omega$

Figure 17: Effect of mutual coupling on RCS of series-fed linear array. $\theta_{s}=0^{\circ}, \psi=\pi / 2, d=0.1 \lambda, l=0.5 \lambda, a=10^{-5} \lambda$, and $Z_{0}=150 \Omega$ with unit amplitude uniform distribution for side-by-side configuration.

obtained is in accordance with the results obtained in open literature [6]. Figure 24 shows the similar comparison of the short and impedance terminated feed network but for a scan angle of $50^{\circ}$. A similar trend is observed. This indicates that the termination of the coupler port with a suitable load will reduce the RCS irrespective of the scan angle.

\section{Summary}

This paper presents the study carried out on the estimation of RCS of series-fed linear dipole array in the presence of mutual coupling. Here a step-by-step approach is followed to arrive at the equation of RCS for a phased array with the inclusion of mutual coupling factor. The formulation of the coupling coefficients is first derived in terms of impedance matrix. This is followed by the inclusion of (i) length of the dipole, (ii) effective length of the dipole, and (iii) antenna impedance/radius of the dipole wire into the
RCS equation. The summation term of the scattered field due to the terminating load is refined. This work further presents a detailed formulation for the scattered field at each stage of the feed network. The simulation results are presented for side-by-side, collinear, and parallel-in-echelon configurations of linear dipole array. The RCS pattern obtained in the presence of mutual coupling is compared with no mutual coupling case. The authenticity of the results is established by comparing them with the trends, inferences, and results reported in the open domain.

It is shown that the mutual coupling alters the impedance at the terminals of the radiating elements significantly. The observed changes depend upon the configuration of dipole array considered, namely, side-by-side configuration, collinear configuration, or parallel-in-echelon configuration. The simulation results indicate that the mutual coupling affects the RCS pattern pronouncedly for large scan angles. It is seen that the RCS value can be reduced by varying certain 


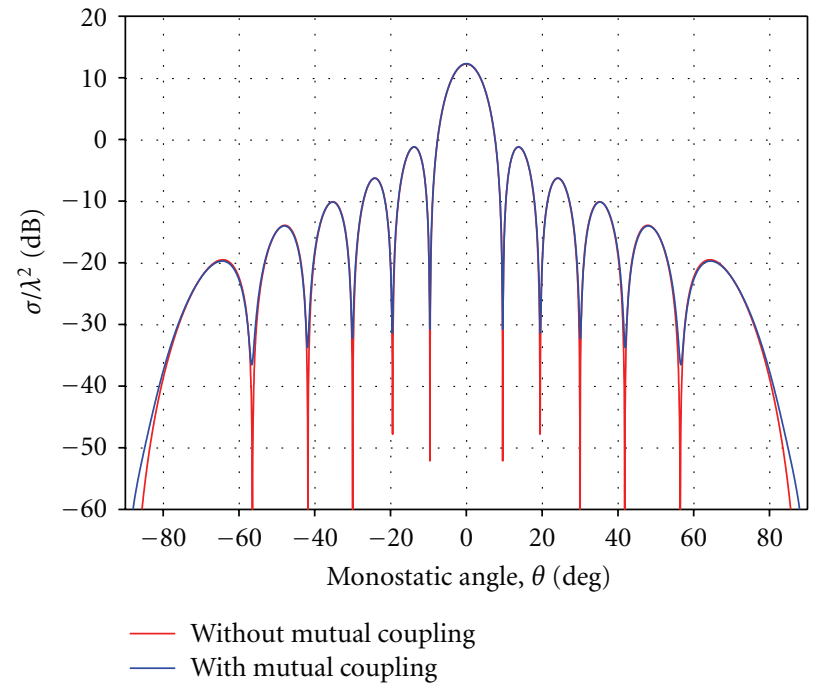

(a) $\theta_{s}=0^{\circ}$

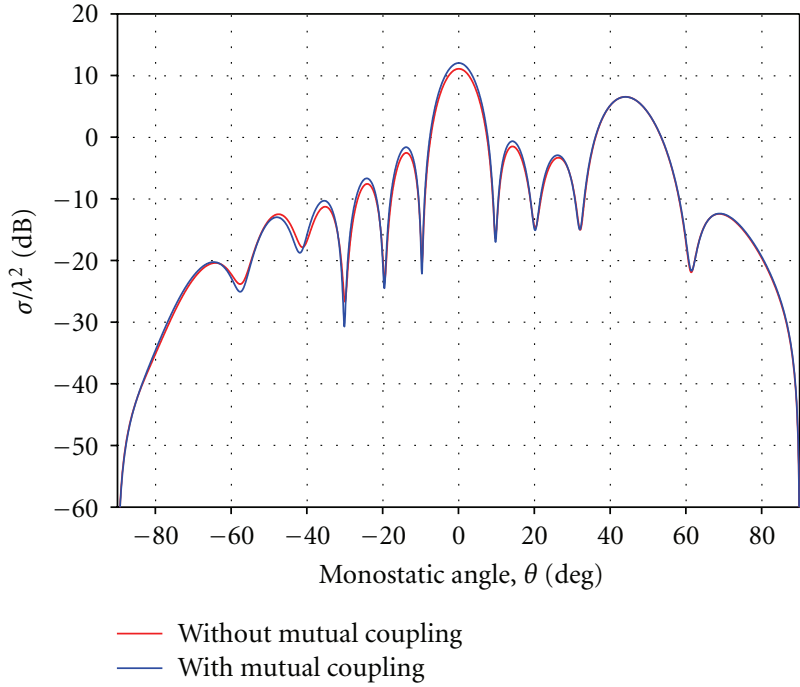

(b) $\theta_{s}=45^{\circ}$

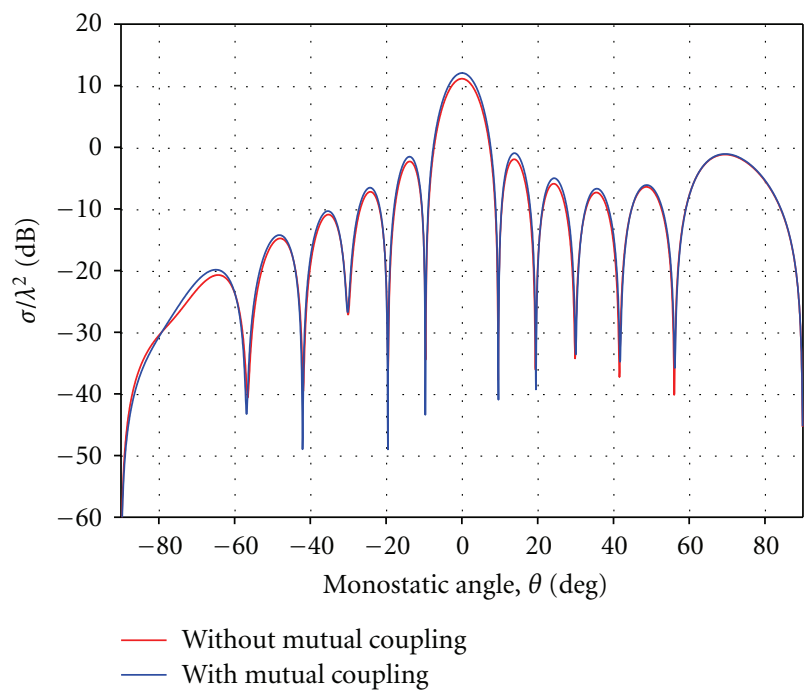

(c) $\theta_{s}=85^{\circ}$

FigURE 18: Effect of mutual coupling on RCS of series-fed linear collinear dipole array of $N=30, \psi=\pi / 2, d=0.1 \lambda, l=0.5 \lambda, a=$ $10^{-5} \lambda, Z_{0}=75 \Omega$, and $Z_{l}=150 \Omega$; unit amplitude uniform distribution.

feed network parameters. It is inferred that the RCS of the dipole array can be reduced by (i) reducing the length of the dipole, (ii) terminating the isolated port of the coupler with a suitable load, or (iii) choosing a suitable amplitude distribution.

$$
\begin{aligned}
& +\frac{1}{2} \cos \left(k l_{n}\right) \\
& \left.\times\left\{C+\ln \left(\frac{k l_{n}}{2}\right)+C_{i}\left(2 k l_{n}\right)-2 C_{i}\left(k l_{n}\right)\right\}\right],
\end{aligned}
$$

\section{Appendices}

\section{A. Self- and Mutual Impedance of Dipole Array}

$$
\begin{aligned}
X_{\text {self }_{n}=\frac{\eta}{4 \pi}[2} & S_{i}\left(k l_{n}\right)+\cos \left(k l_{n}\right)\left\{2 S_{i}\left(k l_{n}\right)-S_{i}\left(2 k l_{n}\right)\right\} \\
& -\sin \left(k l_{n}\right) \\
& \left.\times\left\{2 C_{i}\left(k l_{n}\right)-C_{i}\left(2 k l_{n}\right)-C_{i}\left(\frac{2 k a_{n}^{2}}{l_{n}}\right)\right\}\right],
\end{aligned}
$$$$
R_{\text {self }_{n}}=\frac{\eta}{2 \pi}\left[C+\ln \left(k l_{n}\right)-C_{i}\left(k l_{n}\right)\right.
$$$$
+\frac{1}{2} \sin \left(k l_{n}\right)\left\{S_{i}\left(2 k l_{n}\right)-2 S_{i}\left(k l_{n}\right)\right\}
$$ 


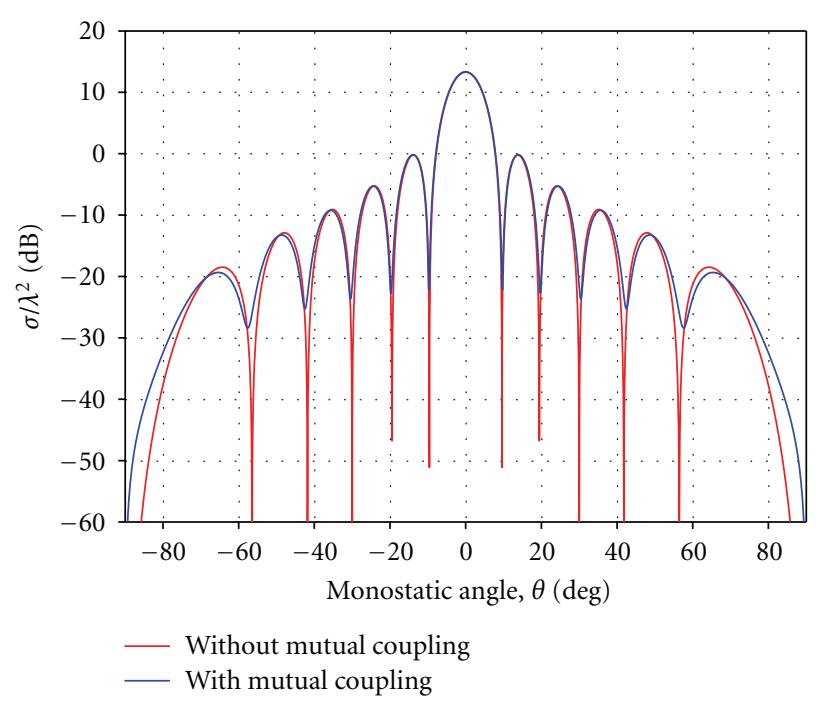

(a) $\theta_{s}=0^{\circ}$

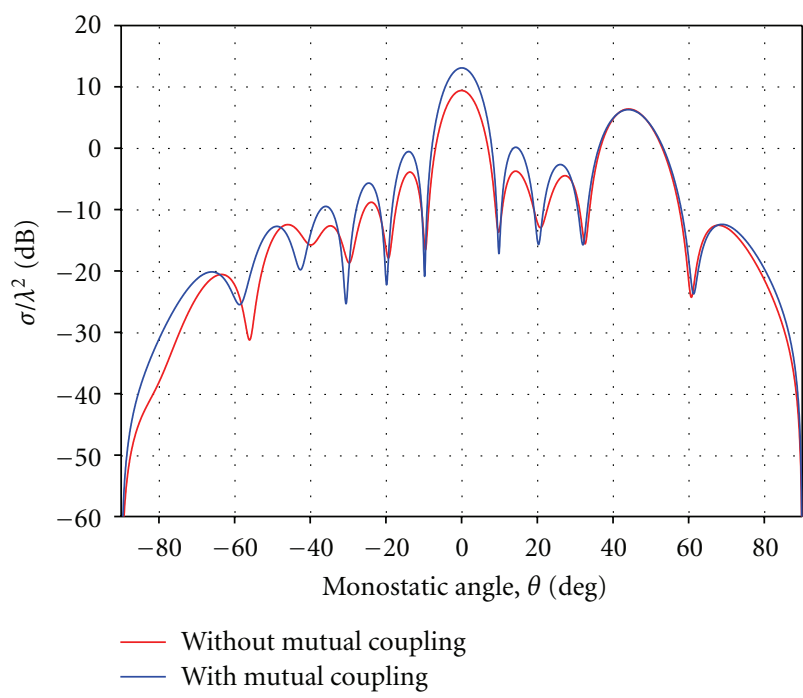

(b) $\theta_{s}=45^{\circ}$

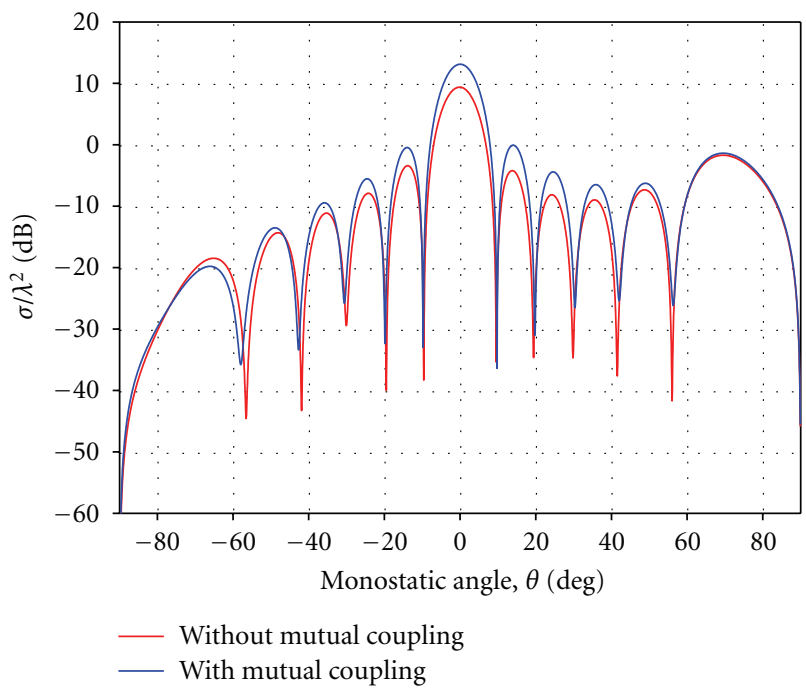

(c) $\theta_{s}=85^{\circ}$

FIGURE 19: Effect of mutual coupling on RCS of series-fed linear parallel-in-echelon dipole array of $N=30, \psi=\pi / 2, d=0.1 \lambda, l=0.5 \lambda, a=$ $10^{-5} \lambda, Z_{0}=125 \Omega$, and $Z_{l}=235 \Omega$; unit amplitude uniform distribution.

where $C_{i}\left(k l_{n}\right)$ and $S_{i}\left(k l_{n}\right)$ are cosine and sine integrals expressed as [5]

$$
\begin{gathered}
S_{i}(x)=\sum_{k=0}^{\infty} \frac{(-1)^{k} x^{2 k+1}}{(2 k+1)(2 k+1) !} \\
C_{i}(x)=C+\ln (x)+\sum_{k=1}^{\infty}(-1)^{k} \frac{x^{2 k}}{2 k(2 k) !} .
\end{gathered}
$$

The expressions of the mutual impedances for different configurations are [5] the following.

Side-by-side configuration:

$$
\begin{aligned}
& R_{s_{r}}=\frac{\eta}{4 \pi}\left[2 C_{i}\left(u_{0_{r}}\right)-C_{i}\left(u_{1_{r}}\right)-C_{i}\left(u_{2_{r}}\right)\right], \\
& X_{s_{r}}=-\frac{\eta}{4 \pi}\left[2 S_{i}\left(u_{0_{r}}\right)-S_{i}\left(u_{1_{r}}\right)-S_{i}\left(u_{2_{r}}\right)\right],
\end{aligned}
$$

where $u_{0_{r}}=k d_{r} ; u_{1_{r}}=k\left(\sqrt{d_{r}^{2}+l_{n}^{2}}+l_{n}\right) ; u_{2_{r}}=k\left(\sqrt{d_{r}^{2}+l_{n}^{2}}-l_{n}\right)$.

Collinear configuration:

$$
\begin{aligned}
R_{c_{r}=} & -\frac{\eta}{8 \pi} \cos \left(v_{0_{r}}\right)\left[-2 C_{i}\left(2 v_{0_{r}}\right)+C_{i}\left(v_{2_{r}}\right)+C_{i}\left(v_{1_{r}}\right)\right. \\
& \left.-\ln \left(v_{3_{r}}\right)\right] \\
& +\frac{\eta}{8 \pi} \sin \left(v_{0_{r}}\right)\left[2 S_{i}\left(2 v_{0_{r}}\right)-S_{i}\left(v_{2_{r}}\right)-S_{i}\left(v_{1_{r}}\right)\right],
\end{aligned}
$$

$$
\begin{gathered}
X_{c_{r}=}-\frac{\eta}{8 \pi} \cos \left(v_{0_{r}}\right)\left[2 S_{i}\left(2 v_{0_{r}}\right)-S_{i}\left(v_{2_{r}}\right)-S_{i}\left(v_{1_{r}}\right)\right] \\
+\frac{\eta}{8 \pi} \sin \left(v_{0_{r}}\right)\left[2 C_{i}\left(2 v_{0_{r}}\right)-C_{i}\left(v_{2_{r}}\right)-C_{i}\left(v_{1_{r}}\right)\right. \\
\left.-\ln \left(v_{3_{r}}\right)\right],
\end{gathered}
$$

where $v_{0_{r}}=k\left(d+l_{n}\right) ; v_{1_{r}}=2 k\left(d+2 l_{n}\right) ; v_{2_{r}}=2 k d ; v_{3_{r}}=$ $\left(d^{2}+2 d l_{n}\right) /\left(d+l_{n}\right)^{2}$. 


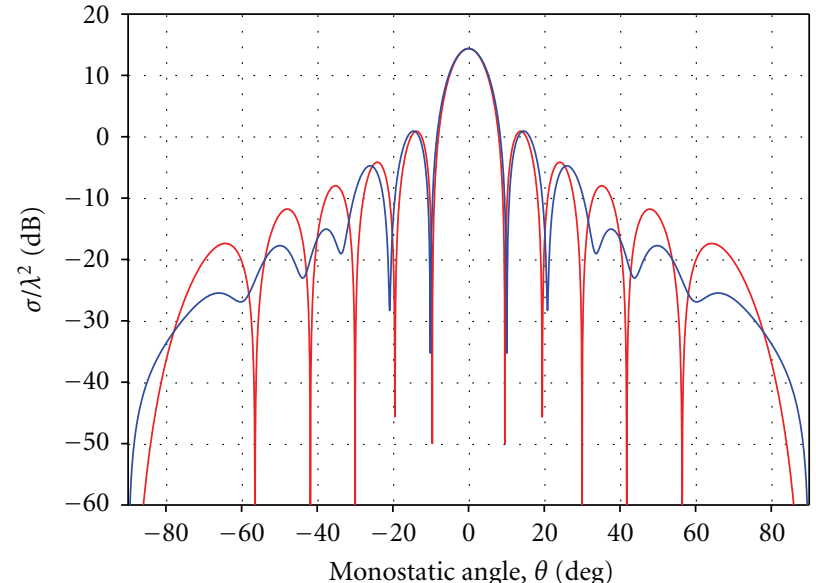

- Without mutual coupling

With mutual coupling

(a) $\theta_{s}=0^{\circ}$

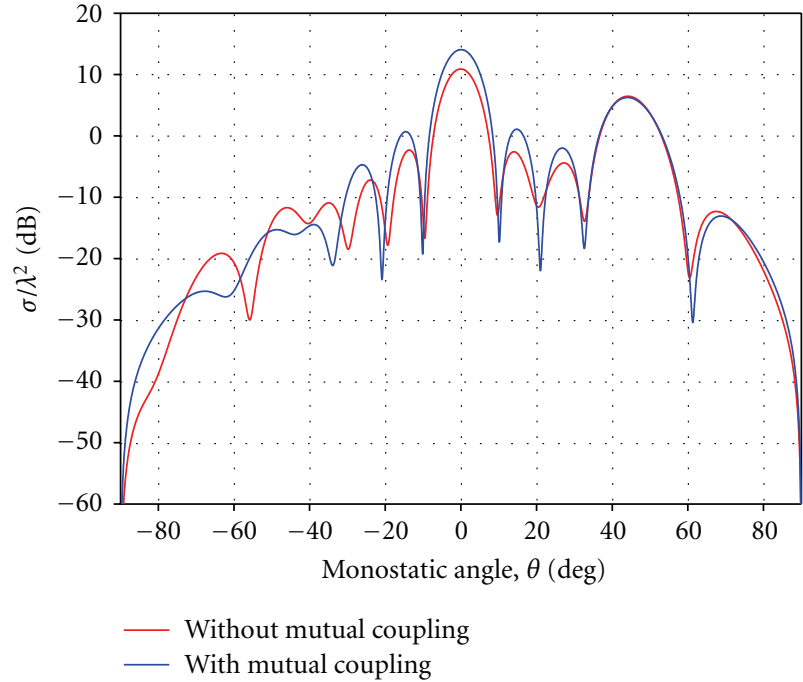

(b) $\theta_{s}=45^{\circ}$

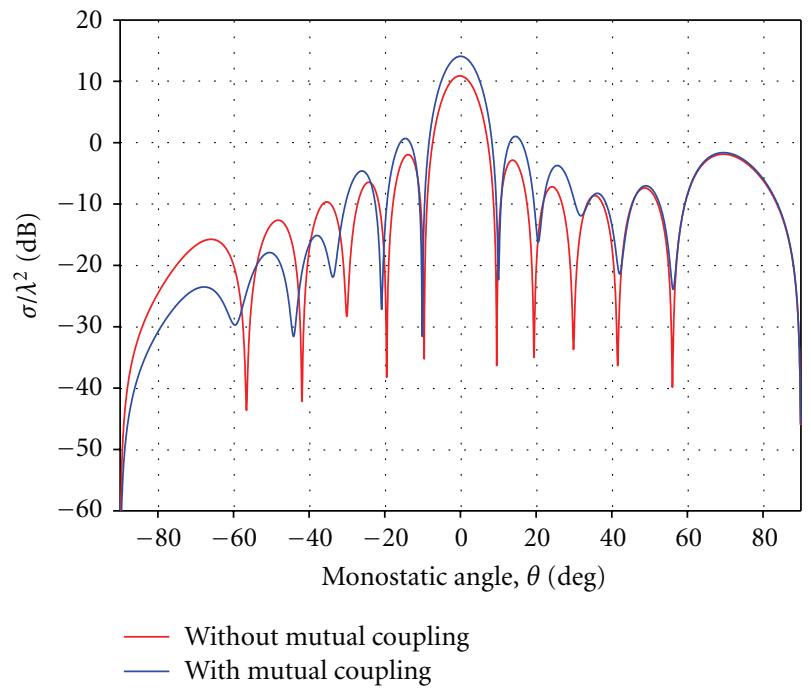

(c) $\theta_{s}=85^{\circ}$

FIgURE 20: Effect of mutual coupling on RCS of series-fed linear side-by-side dipole array of $N=30, \psi=\pi / 2, d=0.1 \lambda, l=0.5 \lambda, a=$ $10^{-5} \lambda, Z_{0}=150 \Omega$, and $Z_{l}=280 \Omega$; unit amplitude uniform distribution.

Parallel-in-echelon configuration:

$$
\begin{aligned}
& R_{p_{r}}=-\frac{\eta}{8 \pi} \cos \left(w_{0_{r}}\right) \\
& \times\left[-2 C_{i}\left(w_{1_{r}}\right)-2 C_{i}\left(w_{1_{r}}^{\prime}\right)\right. \\
&\left.\quad+C_{i}\left(w_{2_{r}}\right)+C_{i}\left(w_{2_{r}}^{\prime}\right)+C_{i}\left(w_{3_{r}}\right)+C_{i}\left(w_{3_{r}}^{\prime}\right)\right] \\
&+\frac{\eta}{8 \pi} \sin \left(w_{0_{r}}\right)\left[2 S_{i}\left(w_{1_{r}}\right)-2 S_{i}\left(w_{1_{r}}^{\prime}\right)-S_{i}\left(w_{2_{r}}\right)\right. \\
&\left.+S_{i}\left(w_{2_{r}}^{\prime}\right)-S_{i}\left(w_{3_{r}}\right)+S_{i}\left(w_{3_{r}}^{\prime}\right)\right],
\end{aligned}
$$

$$
\begin{aligned}
X_{p_{r}}=-\frac{\eta}{8 \pi} \cos \left(w_{0_{r}}\right)[ & 2 S_{i}\left(w_{1_{r}}\right)+2 S_{i}\left(w_{1_{r}}^{\prime}\right)-S_{i}\left(w_{2_{r}}\right) \\
& \left.-S_{i}\left(w_{2_{r}}^{\prime}\right)-S_{i}\left(w_{3_{r}}\right)-S_{i}\left(w_{3_{r}}^{\prime}\right)\right]
\end{aligned}
$$

$$
\begin{aligned}
+\frac{\eta}{8 \pi} \sin \left(w_{0_{r}}\right)[ & 2 C_{i}\left(w_{1_{r}}\right)-2 C_{i}\left(w_{1_{r}}^{\prime}\right)-C_{i}\left(w_{2_{r}}\right) \\
& \left.+C_{i}\left(w_{2_{r}}^{\prime}\right)-C_{i}\left(w_{3_{r}}\right)+C_{i}\left(w_{3_{r}}^{\prime}\right)\right],
\end{aligned}
$$

where $w_{0_{r}}=k h_{r} ; w_{1_{r}}=k\left(\sqrt{d^{2}+h_{r}^{2}}+h_{r}\right) ; w_{1_{r}}^{\prime}=$ $k\left(\sqrt{d^{2}+h_{r}^{2}}-h_{r}\right), w_{2_{r}}=k\left(\sqrt{d^{2}+\left(h_{r}-l_{n}\right)^{2}}+\left(h_{r}-l_{n}\right)\right) ; w_{2_{r}}^{\prime}=$ $k\left(\sqrt{d^{2}+\left(h_{r}-l_{n}\right)^{2}}-\left(h_{r}-l_{n}\right)\right), w_{3_{r}}=k\left(\sqrt{d^{2}+\left(h_{r}+l_{n}\right)^{2}}+\right.$ $\left.\left(h_{r}+l_{n}\right)\right) ; w_{3_{r}}^{\prime}=k\left(\sqrt{d^{2}+\left(h_{r}+l_{n}\right)^{2}}-\left(h_{r}+l_{n}\right)\right)$.

The equations (A.1) through (A.9) are valid for the dipoles of equal length $l$ with identical wire radius $a$. Thus $l_{n}=l$ and $a_{n}=a$ for all $n$ elements of the array. However $d_{r}$ represents the relative distance between the pair of elements considered, $h_{r}$ represents the offset distance between the 


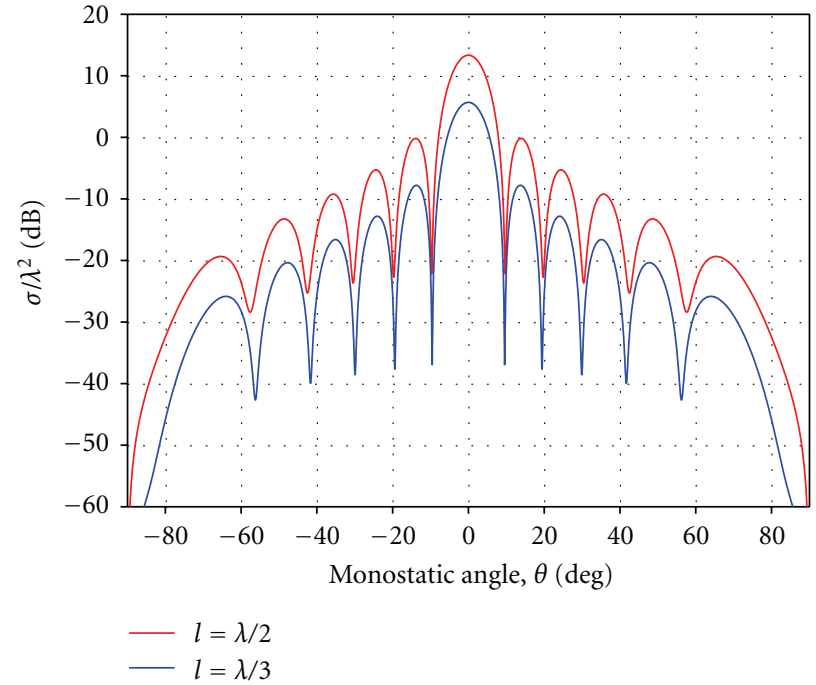

(a) $\theta_{s}=0^{\circ}$

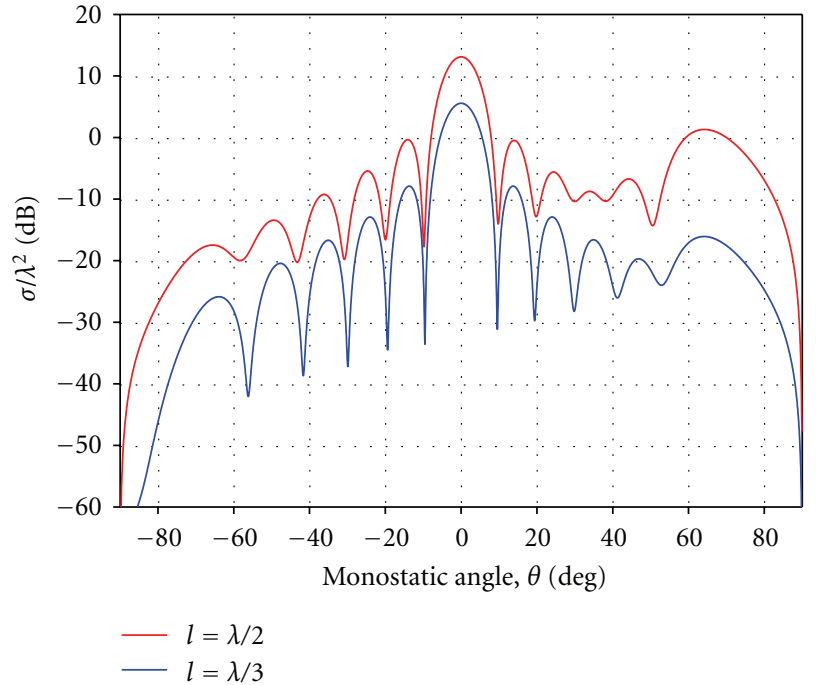

(b) $\theta_{s}=70^{\circ}$

FIGURE 21: Effect of dipole length on RCS of 30-element series-fed linear parallel-in-echelon dipole array including mutual coupling effect.

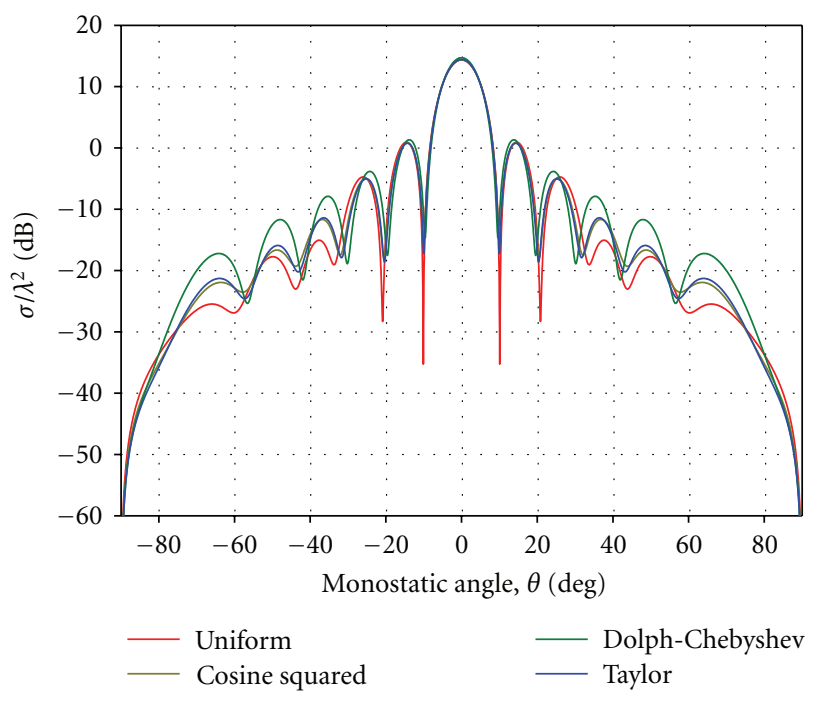

FIGURE 22: Effect of amplitude distribution on RCS of 30-element series-fed linear side-by-side dipole array including mutual coupling effect.

elements under consideration, and $r=(x, y)$ indicates the coordinate pair representation of these elements.

\section{B. Coupling and Transmission Coefficients: Formulation}

The coupling coefficient for a coupler is the ratio of amount of power coupled into the coupling port to the amount of power at its input or receiving port. Thus we have

coupling coefficient $=\frac{\text { Power at the coupling port }}{\text { Power at its receiving port }}$

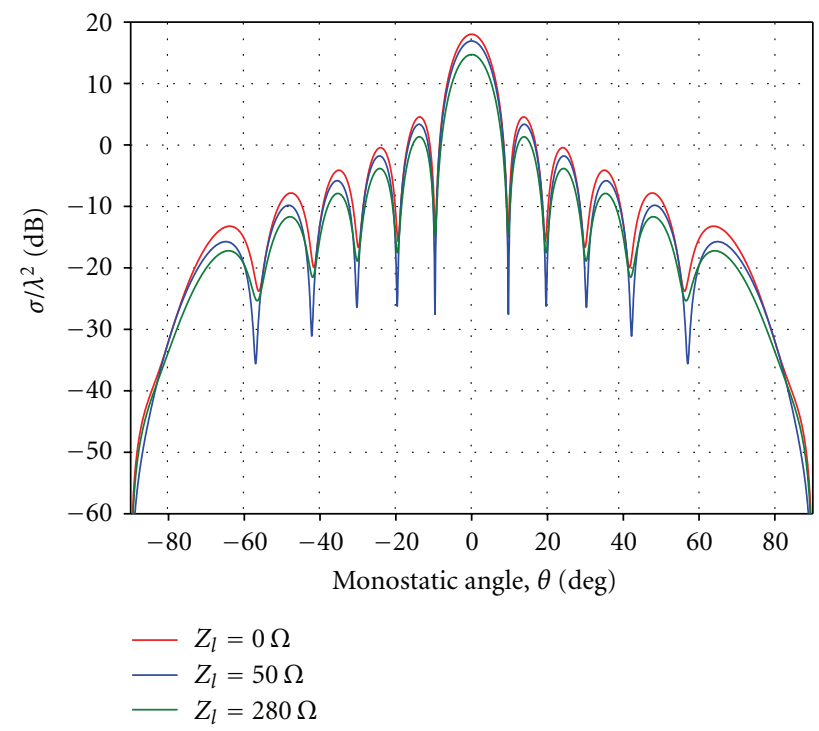

FIGURE 23: Effect of terminating impedance on RCS of series-fed linear array including mutual coupling effect. $N=30, " \theta_{s}=0^{\circ}$ ", $\psi=\pi / 2, l=0.5 \lambda, d=0.1 \lambda, a=10^{-5} \lambda$, and $Z_{0}=150 \Omega$ with Dolph-Chebyshev distribution for side-by-side configuration.

For each coupler in the network, the power at its coupling port will be proportional to the product of current and the impedance at its terminals. However the power at the receiving port of the $n$th coupler will be the total received power minus the power received due to the elements 1 to $n-1$ of the array.

The expression for the coupling coefficient of first element is given by

$$
c_{1}=\frac{P_{1}}{P_{r x}} .
$$




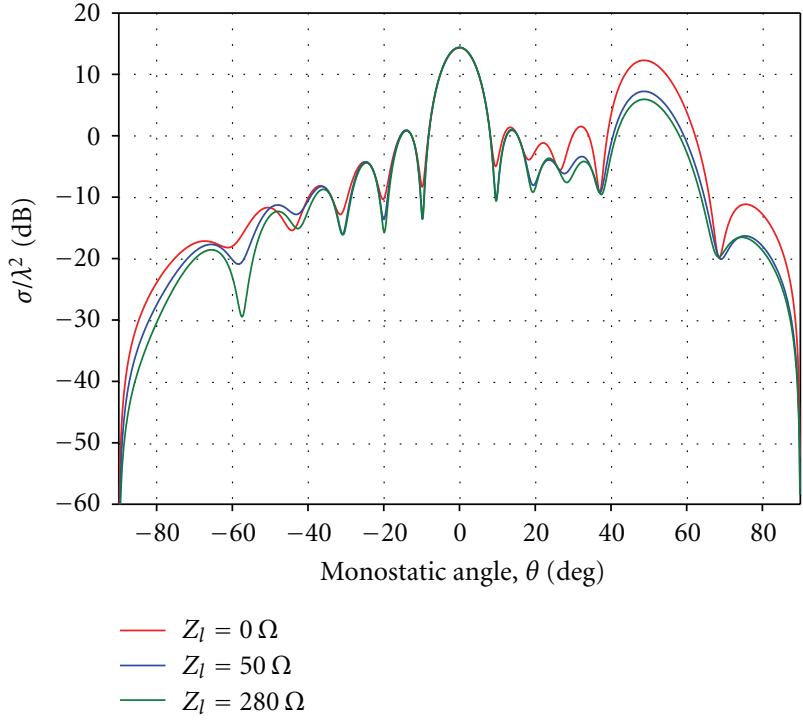

FIGURE 24: Effect of terminating impedance on RCS of series-fed linear array including mutual coupling effect. $N=30$, " $\theta_{s}=50^{\circ}$ ", $\psi=\pi / 2, l=0.5 \lambda, d=0.1 \lambda, a=10^{-5} \lambda$, and $Z_{0}=150 \Omega$ with Dolph-Chebyshev distribution for side-by-side configuration.

Similarly, for other elements we have

$$
c_{2}=\frac{P_{2}}{P_{r x}-P_{1}}=\frac{P_{2}}{P_{r x}-\sum_{q=1}^{1} P_{q}}
$$

(coupling coefficient of the second element)

$$
c_{3}=\frac{P_{3}}{P_{r x}-\left(P_{1}+P_{2}\right)}=\frac{P_{3}}{P_{r x}-\sum_{q=1}^{2} P_{q}}
$$

(coupling coefficient of the third element).

In general,

$$
c_{n}=\frac{P_{n}}{P_{r x}-\left(P_{1}+P_{2}+\cdots+P_{n-1}\right)}=\frac{P_{n}}{P_{r x}-\sum_{q=1}^{n-1} P_{q}}
$$

(coupling coefficient of the $n$th element).

For a lossless network shown in Figure 25, the total power fed into the circuit will be equal to the sum of the powers delivered to the individual antenna elements and to the terminating load. If the same array acts as a receiver, then the total power received should be equal to the sum of the power received by the individual antenna elements (assuming that zero power was delivered to the load while transmission). Thus, we have

$$
P_{r x}=P_{1}+P_{2}+\cdots+P_{n}+\cdots+P_{N-1}+P_{N}=\sum_{p=1}^{N} P_{p} .
$$

Expressing power in terms of current and impedance, one gets

$$
\begin{aligned}
P_{r x}= & z_{1} i_{1}^{2}+z_{2} i_{2}^{2}+\cdots+z_{n} i_{n}^{2} \\
& +\cdots+z_{N-1} i_{N-1}^{2}+z_{N} i_{N}^{2}=\sum_{p=1}^{N} z_{p} i_{p}^{2},
\end{aligned}
$$

where $z_{p}$ is the net impedance of the $p$ th antenna element, $i_{p}$ is the current at the terminals of $p$ th antenna element, and $P_{r x}$ is the total received power.

Here $z_{p}$ is given by $z_{p}=\sum_{q=1}^{N} z_{p, q}\left(I_{q} / I_{p}\right)$ [5] with $p$ and $q$ being the row and column numbers of the impedance matrix [26]

$$
z_{p, q}=\left(\begin{array}{cccc}
z_{1,1} & z_{1,2} & \cdots & z_{1, N} \\
z_{2,1} & z_{2,2} & \cdots & z_{2, N} \\
\vdots & \vdots & \ddots & \vdots \\
z_{N, 1} & z_{N, 2} & \cdots & z_{N, N}
\end{array}\right) .
$$

Thus the coupling coefficient of couplers can be written as

$$
c_{n}=\frac{z_{n} i_{n}^{2}}{\sum_{p=1}^{N} z_{p} i_{p}^{2}-\sum_{q=1}^{n-1} z_{q} i_{q}^{2}},
$$

and the transmission coefficient is given by

$$
t_{c_{n}}^{2}=1-c_{n}^{2}
$$

\section{List of Symbols}

$\alpha: \quad$ Interelement space delay along $x$ direction

$\alpha_{s}$ : Interelement phase to scan antenna beam along $x$ direction

$\Gamma_{n}^{r}$ : Total reflected signal returning to aperture element $n$

$\eta$ : $\quad$ Impedance of medium around antenna

$\eta_{o}: \quad$ Impedance of free space

$(\theta, \phi): \quad$ Direction of incident signal

$\left(\theta_{s}, \phi_{s}\right):$ Beam scan angle

$\lambda$ : Wavelength

$\sigma(\theta, \phi)$ : Overall RCS of phased array

$\sigma_{c}(\theta, \phi)$ : RCS due to scattering from coupling port of the couplers

$\sigma_{p}(\theta, \phi):$ RCS due to scattering from phase shifters

$\sigma_{r}(\theta, \phi)$ : RCS due to scattering from radiators

$\sigma_{s}(\theta, \phi)$ : RCS due to scattering beyond the coupling port of couplers

$a$ : $\quad$ Radius of dipole wire

$c_{n}$ : $\quad$ Coupling coefficient of $n$th coupler

$C: \quad$ Euler's constant

$C_{i}(x): \quad$ Cosine integral

$d: \quad$ Interelement spacing

$\vec{d}_{n}: \quad$ Distance vector

$d_{r}$ : Relative distance between element pair

$\vec{E}^{i}(\theta, \phi)$ : Incident field

$\vec{E}_{c_{n}}^{r}(\theta, \phi)$ : Scattered field due to $n$th coupler

$\vec{E}_{n}^{r}(\theta, \phi)$ : Total scattered field

$\vec{E}_{p_{n}}^{r}(\theta, \phi)$ : Scattered field due to $n$th phase shifter

$\vec{E}_{r_{n}}^{r}(\theta, \phi)$ : Scattered field due to $n$th dipole

$\vec{E}_{s_{n}}^{r}(\theta, \phi)$ : Total scattered field due to the scattering beyond the coupling port of couplers

$\overrightarrow{E^{s}}$ : Total scattered field

$\vec{E}_{n}^{s}$ : $\quad$ Total scattered field due to single element

F: $\quad$ Constant 


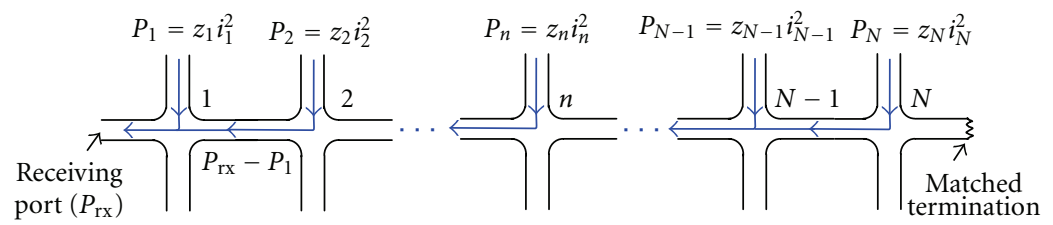

FIgURE 25: Coupling and transmission coefficients of the couplers.

$\vec{h}$ : $\quad$ Effective height of a $x$-polarized antenna element

$i_{n}$ : $\quad$ Current at the terminals of $n$th antenna element

$I(0)$ : Current at the feed terminals of antenna

$I_{n}$ : Current at the feed terminals of $n$th antenna element

$k$ : Wave number

$\vec{k}$ : Wave vector

$l$ : Dipole length

$L_{n}$ : Length of delay lines

$N$ : Number of elements in the antenna array

$P_{n}: \quad$ Power received at the $n$th coupler

$P_{r x}$ : Total received power

$r_{c_{n}}$ : Reflection coefficient at the coupling port of the $n$th coupler

$r_{i n}$ : Reflection coefficient at the receive port of the array

$r_{l_{n}}$ : Reflection coefficient of the load terminating isolation port of $n$th coupler

$r_{p_{n}}$ : Reflection coefficient of $n$th phase shifter

$r_{r_{n}}$ : Reflection coefficient of $n$th radiating element

$R: \quad$ Distance between the target and the observation point

$R_{a}$ : Antenna resistance

$R_{c_{r}}$ : Radiation resistance due to collinear configuration

$R_{d}$ : Loss resistance of antenna

$R_{p_{r}}$ : Radiation resistance due to parallel-in-echelon configuration

$R_{r}$ : Radiation resistance

$R_{s_{r}}$ : Radiation resistance due to side-by-side configuration

$R_{\text {self }_{n}}$ : Self-coupling radiation resistance of $n$th dipole

$S_{i}(x)$ : Sine integral

$t_{c_{n}}$ : Transmission coefficient of $n$th coupler

$t_{p_{n}}$ : Transmission coefficient of $n$th phase shifter

$t_{r_{n}}$ : Transmission coefficient of $n$ th-radiating element

$X_{a}$ : Antenna reactance

$X_{c_{r}}$ : Radiation reactance due to collinear configuration

$X_{p_{r}}$ : Radiation reactance due to parallel-in-echelon configuration

$X_{s_{r}}$ : Radiation reactance due to side-by-side configuration

$X_{\text {self }_{n}}$ : Radiation reactance of $n$th antenna element due to self-coupling $z_{a_{x, y}}$ : Mutual impedance between $x$ th and $y$ th dipoles

$z_{p}$ : Net impedance at $p$ th antenna element

$Z_{12}$ : Impedance at the input port of the first coupler in the array

$Z_{0}$ : Characteristic impedance

$Z_{a}$ : Antenna impedance

$Z_{a_{n}}$ : Terminal impedance of $n$th antenna element

$Z_{p_{n}}$ : Terminal impedance of $n$th phase shifter.

\section{Acknowledgment}

This work was funded as a sponsored project at CSIRNational Aerospace Laboratories, Bangalore, India by Aeronautics Research and Development Board (AR\&DB), New Delhi, India.

\section{References}

[1] E. F. Knott, J. F. Shaeffer, and M. T. Tuley, Radar Cross Section, Artech House, Dedham, Mass, USA, 1985.

[2] D. C. Jenn, Radar and Laser Cross Section Engineering, AIAA Education Series, Washington, DC, USA, 1995.

[3] J. L. Volakis, Antenna Engineering Handbook, McGraw Hill, New York, NY, USA, 4th edition, 2007.

[4] S. Zhang, S. X. Gong, Y. Guan, and B. Lu, "Optimized element positions for prescribed radar cross section pattern of linear dipole arrays," International Journal of RF and Microwave Computer-Aided Engineering, vol. 21, pp. 622-628, 2011.

[5] C. A. Balanis, Antenna Theory, Analysis and Design, John Wiley \& Sons, Hoboken, NJ, USA, 2005.

[6] L. Zengrui, W. Junhong, L. Limei, and Z. Xueqin, "Study on the scattering property of the impedance terminated dipole array with finite reflector by FDTD method," in Proceedings of the IEEE International Symposium on Microwave, Antenna, Propagation, and EMC Technologies for Wireless Communications (MAPE '07), pp. 1003-1007, Hangzhou, China, August 2007.

[7] F. Dikmen, A. A. Ergin, A. L. Sevgili, and B. Terzi, "Implementation of an efficient shooting and bouncing rays scheme," Microwave and Optical Technology Letters, vol. 52, no. 11, pp. 2409-2413, 2010.

[8] Y. Tao, H. Lin, and H. Bao, "GPU-based shooting and bouncing ray method for fast RCS prediction," IEEE Transactions on Antennas and Propagation, vol. 58, no. 2, pp. 494-502, 2010.

[9] A. Zdunek and W. Rachowicz, "Cavity radar cross section prediction," IEEE Transactions on Antennas and Propagation, vol. 56, no. 6, pp. 1752-1762, 2008.

[10] K. K. Chan and K. Chadwick, "Computation of radar cross section of waveguide arrays," in Proceedings of IEEE Antennas and Propagation Society International Symposium, pp. 17461749, Orlando, Fla, USA, July 1999. 
[11] P. J. Tittensor and M. L. Newton, "Prediction of the radar cross-section of an array antenna," in Proceedings of the 6th International Conference on Antennas and Propagation (ICAP '89), pp. 258-262, April 1989.

[12] W. T. Wang, Y. Liu, S. X. Gong, Y. J. Zhang, and X. Wang, "Calculation of antenna mode scattering based on method of moments," Progress in Electromagnetics Research Letters, vol. 15, pp. 117-126, 2010.

[13] S. Zhang, S. X. Gong, Y. Guan, J. Ling, and B. Lu, "A new approach for synthesizing both the radiation and scattering patterns of linear dipole antenna array," Journal of Electromagnetic Waves and Applications, vol. 24, no. 7, pp. 861-870, 2010.

[14] W. T. Wang, S. X. Gong, Y. J. Zhang, F. T. Zha, J. Ling, and T. T. Wan, "Low RCS dipole array synthesis based on MoM-PSO hybrid algorithm," Progress in Electromagnetics Research, vol. 94, pp. 119-132, 2009.

[15] H. T. Hui, "Decoupling methods for the mutual coupling effect in antenna arrays: a review," Recent Patents on Engineering, vol. 1, pp. 187-193, 2007.

[16] I. J. Gupta and A. A. Ksienski, "Effect of mutual coupling on the performance of adaptive arrays," IEEE Transactions on Antennas and Propagation, vol. 31, no. 5, pp. 785-791, 1983.

[17] H. T. Hui, "A practical approach to compensate for the mutual coupling effect in an adaptive dipole array," IEEE Transactions on Antennas and Propagation, vol. 52, no. 5, pp. 1262-1269, 2004.

[18] Y. Yu and H. T. Hui, "Design of a mutual coupling compensation network for a small receiving monopole array," IEEE Transactions on Microwave Theory and Techniques, vol. 59, no. 9, pp. 2241-2245, 2011.

[19] K. C. Lee and T. H. Chu, "Mutual coupling mechanisms within arrays of nonlinear antennas," IEEE Transactions on Electromagnetic Compatibility, vol. 47, no. 4, pp. 963-970, 2005.

[20] B. Lu, S. X. Gong, S. Zhang, and J. Ling, "A new method for determining the scattering of linear polarized element arrays," Progress in Electromagnetics Research M, vol. 7, pp. 87-96, 2009.

[21] D. C. Jenn and S. Lee, "Inband scattering from arrays with series feed networks," IEEE Transactions on Antennas and Propagation, vol. 43, no. 8, pp. 867-873, 1995.

[22] K. M. Lee and R. S. Chu, "Analysis of mutual coupling between a finite phased array of dipoles and its feed network," IEEE Transactions on Antennas and Propagation, vol. 36, no. 12, pp. 1681-1699, 1988.

[23] F. Najib, R. Mohamad, and V. Patrick, "Effects of mutual coupling in phased arrays with butler network feed," in Proceedings of 17th International Conference on Applied Electromagnetics and Communications, pp. 374-379, Dubrovnik, Croatia, October 2003.

[24] H. M. Aumann, A. J. Fenn, and F. G. Willwerth, "Phased array antenna calibration and pattern prediction using mutual coupling measurements," IEEE Transactions on Antennas and Propagation, vol. 37, no. 7, pp. 844-850, 1989.

[25] R. S. Chu, "Analysis of an infinite phased array of dipole elements with RAM coating on ground plane and covered with layered radome," IEEE Transactions on Antennas and Propagation, vol. 39, no. 2, pp. 164-176, 1991.

[26] I. J. Gupta and A. A. Ksienski, "Prediction of adaptive array performance," IEEE Transactions on Aerospace and Electronic Systems, vol. 19, no. 3, pp. 380-388, 1983.

[27] J. Ehmouda, Z. Briqech, and A. Amer, "Steered microstrip phased array antennas," Proceedings of World Academy of
Science, Engineering and Technology, vol. 37, pp. 319-323, 2009.

[28] S. Y. Liao, Microwave Devices and Circuits, Prentice Hall, Englewood Cliffs, NJ, USA, 1990.

[29] H. Singh, H. L. Sneha, and R. M. Jha, "Estimation of inband radar cross section (RCS) of phased array with seriesfeed network," Project Document PD AL 1109, CSIR-National Aerospace Laboratories, Bangalore, India, 2011.

[30] S. Zhang, S. X. Gong, and P. F. Zhang, "A modified PSO for low sidelobe concentric ring arrays synthesis with multiple constraints," Journal of Electromagnetic Waves and Applications, vol. 23, no. 11-12, pp. 1535-1544, 2009.

[31] S. Zhang, S. X. Gong, Y. Guan, and B. Lu, "A novel approach for reducing the computational cost of GA in synthesizing low sidelobe unequally spaced linear arrays," International Journal of RF and Microwave Computer-Aided Engineering, vol. 21, no. 2, pp. 221-227, 2011.

[32] B. Lu, S. X. Gong, S. Zhang, Y. Guan, and J. Ling, "Optimum spatial arrangement of array elements for suppression of grating-lobes of radar cross section," IEEE Antennas and Wireless Propagation Letters, vol. 9, pp. 114-117, 2010. 

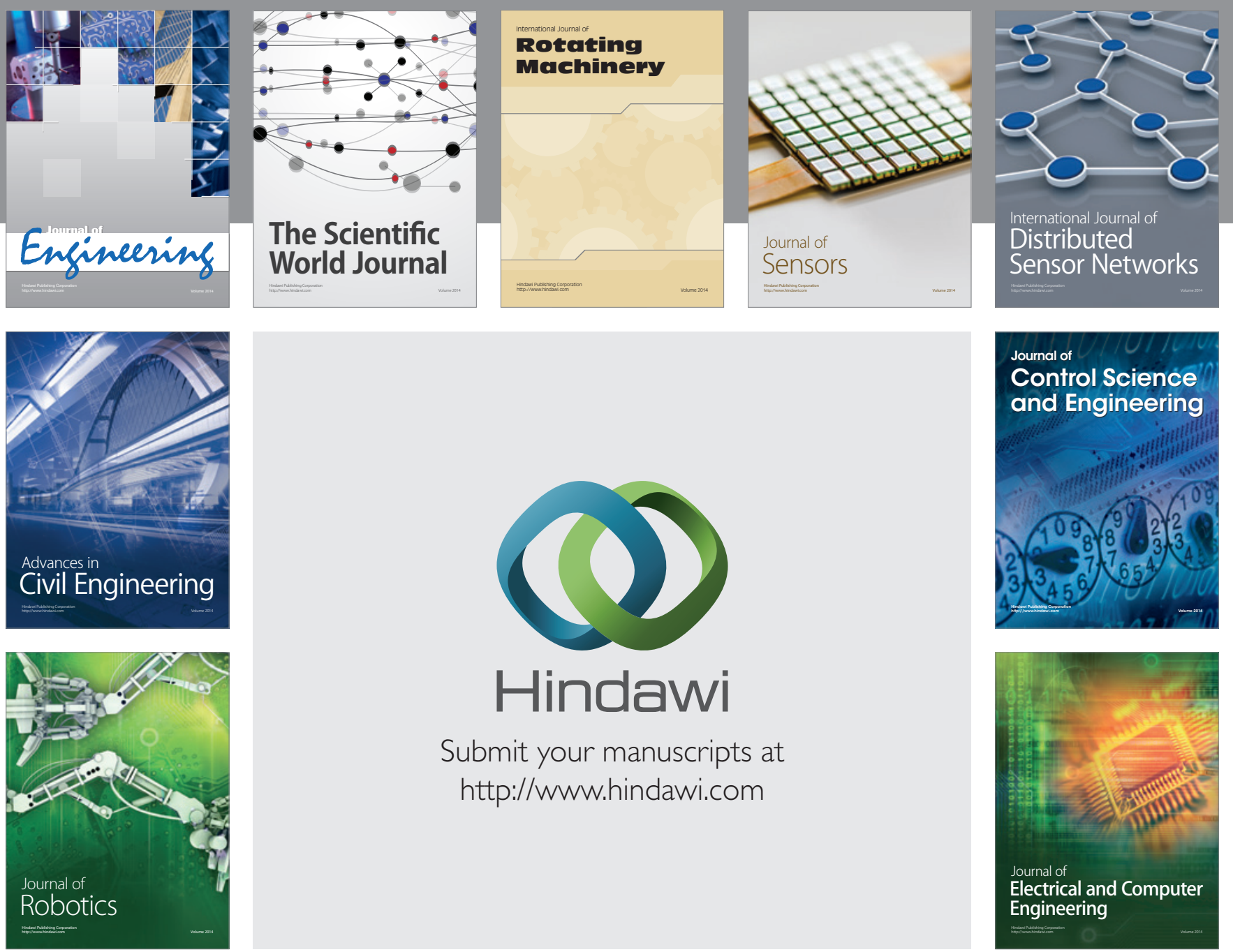

Submit your manuscripts at

http://www.hindawi.com
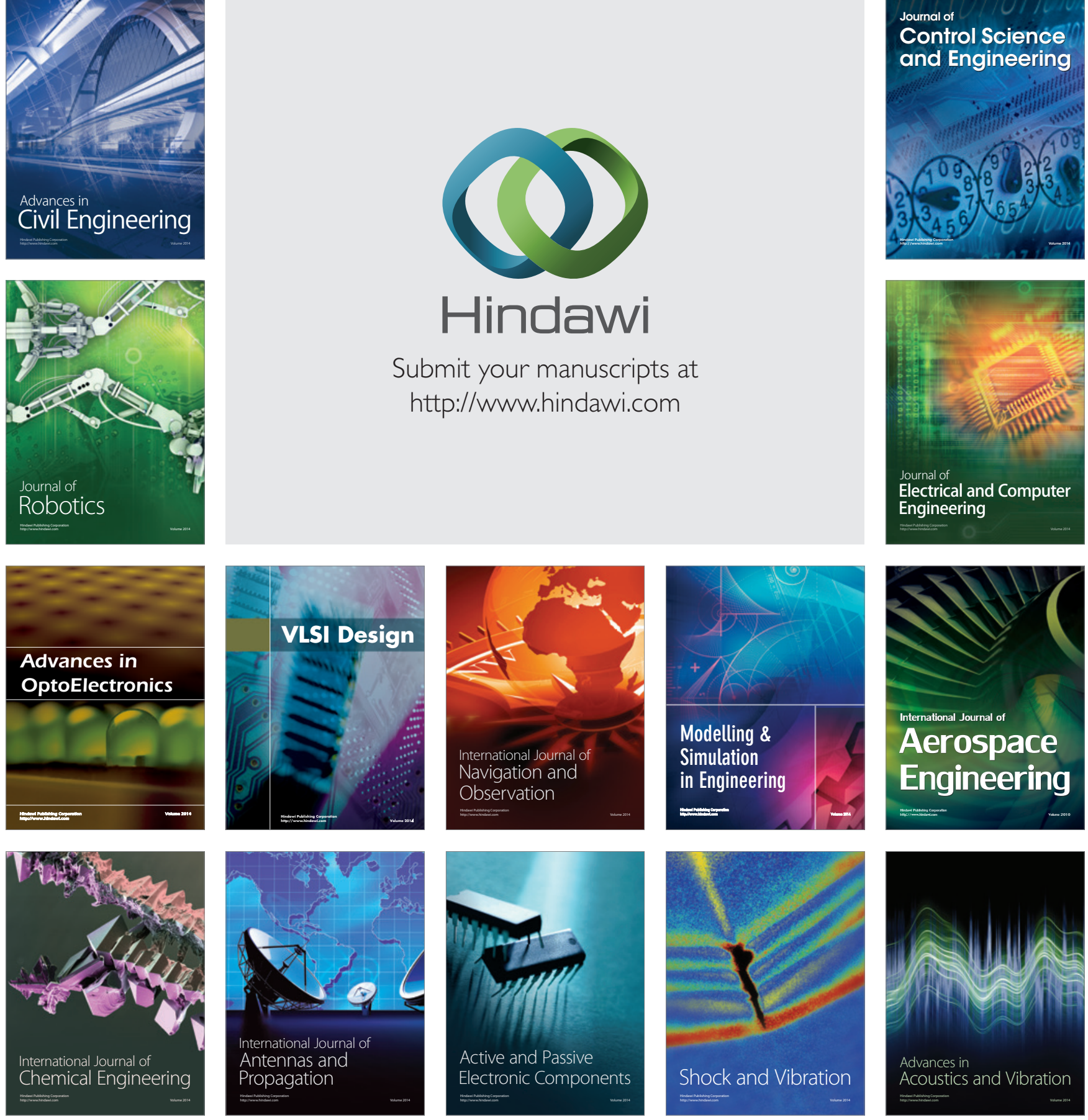